\title{
Reflectance and transmittance model for recto-verso halftone prints
}

\author{
Mathieu Hébert and Roger David Hersch \\ Ecole Polytechnique Fédérale de Lausanne, Switzerland 1015
}

Received January 17, 2006; accepted April 9, 2006; posted May 5, 2006 (Doc. ID 67306)

\begin{abstract}
We propose a spectral prediction model for predicting the reflectance and transmittance of recto-verso halftone prints. A recto-verso halftone print is modeled as a diffusing substrate surrounded by two inked interfaces in contact with air (or with another medium). The interaction of light with the print comprises three components: (a) the attenuation of the incident light penetrating the print across the inked interface, (b) the internal reflectance and internal transmittance that accounts for the substrate's intrinsic reflectance and transmittance and for the multiple Fresnel internal reflections at the inked interfaces, and (c) the attenuation of light exiting the print across the inked interfaces. Both the classical Williams-Clapper and Clapper-Yule spectral prediction models are special cases of the proposed recto-verso reflectance and transmittance model. We also extend the Kubelka-Munk model to predict the reflectance and transmittance of recto-verso halftone prints. The extended Kubelka-Munk model is compatible with the proposed recto-verso reflectance and transmittance model. In the case of a homogeneous substrate, the recto-verso model's internal reflectance and transmittance can be expressed as a function Kubelka-Munk's scattering and absorption parameters, or the Kubelka-Munk's scattering and absorption parameters can be inferred from the recto-verso model's internal reflectance and transmittance, deduced from spectral measurements. The proposed model offers new perspectives both for spectral transmission and reflection predictions and for characterizing the properties of printed diffuse substrates. (C) 2006 Optical Society of America
\end{abstract}

OCIS codes: $100.2810,120.5700,120.7000$.

\section{INTRODUCTION}

Modeling the interaction of light, ink halftones, and a paper substrate has been a research challenge since the early 1930s. Many different phenomena influence the reflection or transmission spectrum of a color halftone patch printed on a diffusely reflecting or transmitting substrate (e.g., paper). These phenomena comprise (a) the surface reflection of incident light (Fresnel reflectance) at the interface between the air and the paper; (b) scattering, lateral propagation, and reflection of light within the substrate; and (c) the internal reflections (Fresnel reflections) at each interface between the paper and the air. In addition to these purely optical phenomena, the ink interacts with the paper at print time, leading to ink spreading and possibly to the partial penetration of ink within the paper substrate.

Most existing spectral prediction models predict the reflectance but not the transmittance of halftone prints. They also assume that the prints are printed only on their upper side. In this paper we present a spectral prediction model predicting both the spectral reflectance and the transmittance of halftone prints, printed either on one side (the recto) or on two sides (recto-verso) of a printed sheet. We also extend the Kubelka-Munk theory ${ }^{1}$ to substrates printed on one or both sides with ink halftones. We then establish the relationship between the intrinsic reflectance and transmittance coefficients of the proposed reflection and transmission prediction model and the Kubelka-Munk scattering and absorption coefficients.

Early attempts to model the reflectance of a halftone print include the Neugebauer spectral reflectance prediction model, ${ }^{2}$ which neglects both the lateral propagation of light within the paper and the Fresnel reflections at the print-air interface. Yule and Nielsen ${ }^{3}$ and Viggiano ${ }^{4}$ improve the Neugebauer model by applying a power function to the reflectances, which accounts for the lateral propagation of light within the paper and for the internal Fresnel reflections. The Clapper-Yule reflectance model ${ }^{5}$ improves the Neugebauer model by explicitly modeling the internal (Fresnel) reflections at the paper-air interface and by accounting for the lateral propagation of light within the paper. The Williams-Clapper model, ${ }^{6}$ supporting solid ink prints only, accounts explicitly both for the Fresnel reflections at the upper paper-air interface and for the obliqueness of the light rays traversing the ink layer. The Kubelka-Munk model predicts the reflectance and transmittance of a single uniformly diffusing layer substrate. ${ }^{1,7}$ Kubelka $^{8}$ extended the model to nonuniformly diffusing, possibly multilayer, substrates but without accounting for the internal Fresnel reflections at the substrate interface with the air. Saunderson introduces a correction $^{9}$ that computes the exterior reflectance from the Kubelka-Munk reflectance (internal reflectance) model, accounting for multiple internal Fresnel reflections.

More recent reflectance models include probabilistic models of the lateral propagation of light within the substrate but do not consider explicitly the internal Fresnel reflections at the interface between the print and the air. ${ }^{10-12}$ Reflectance models accounting for the internal Fresnel reflections are extensions of the Clapper-Yule model $^{13-15}$ or of the Kubelka-Munk model. ${ }^{16}$ Recent extensions of the Kubelka-Munk model ${ }^{17-19}$ concern absorbing and inhomogeneous scattering media, such as prints 
in which the solid ink partially penetrates into the diffusing substrate.

In this paper we rely on the following assumptions. The recto-verso print is composed of five superposed optical elements with which light behaves differently: one strongly scattering layer (paper substrate) at the center, two different absorbing and nonscattering layers (solid or halftone inked layers) at both sides of the paper layer, and two interfaces. The recto of the recto-verso print is the side illuminated by the light source and the verso is the other side.

Let us present the characteristics of the interfaces, the inked layers, and the substrate. The interfaces are assumed to be flat. They have a relative refractive index $n$, and the ratio of the print's refractive index to the refractive index of air is equal to 1 . The inked layers are nonscattering, nonflorescent, and of constant thickness. They have a spectral transmittance $t(\lambda)$, called normal transmittance, corresponding to the attenuation of light crossing the layer along its normal direction. The superposition of two solid inks yields a new colorant, e.g., the superposition of magenta and yellow inks yields the red colorant. For a halftone inked layer, each contributing ink is deposited as a dot screen covering a given fraction of the print's surface. The inked layer is a juxtaposition of small colorant areas resulting from the superposition of the ink dot screens. Each colorant area $k$ is characterized by its fractional surface coverage $a_{k}$ and its spectral transmittance $t_{k}(\lambda)$. When printing with cyan, magenta, and yellow inks, we obtain the colorants white (no ink); cyan, magenta, yellow, red (magenta+yellow); green (cyan+yellow); blue (cyan+magenta); and black (cyan + magenta+yellow). In analogy with the spectral Neugebauer reflectance model, ${ }^{2,4}$ the average transmittance $t(\lambda)$ of the halftone inked layer for normal incidence is given by the sum of the transmittances $t_{k}(\lambda)$ of the colorants weighted by their respective fractional surface coverages $a_{k}$ :

$$
t(\lambda)=\sum_{k=1}^{8} a_{k} t_{k}(\lambda) .
$$

We assume that the inks do not penetrate the substrate. In analogy with the Clapper-Yule model, ${ }^{5}$ we assume that lateral light propagation within the substrate is important with respect to the halftone screen period. Both the inked layer and the substrate are assumed to have the same refractive index. Since neither the inked layers nor the interfaces with air scatter light, we consider an ink layer with its interface as a single optical element called the colored interface. Because the inks printed on the recto and the verso are generally different, the recto- and verso-colored interfaces are accordingly different. The substrate is strongly scattering and nonfluorescing. It may have different optical properties at its recto and verso sides. An incident light penetrating the substrate is immediately diffused and its initial angular distribution is canceled. Therefore, the optical properties of the substrate alone are independent of the incident angular distribution. When illuminated at its recto side (its verso side), its reflectance is called recto intrinsic reflectance (verso intrinsic reflectance). According to the prin- ciple of nonpolarity of transmittance introduced by Kubelka, ${ }^{8}$ the substrate has a single intrinsic transmittance, this one being independent of the side of illumination. We assume that light emerging from the substrate is uniformly distributed over the hemisphere, i.e., it is Lambertian.

Let us now consider the superposition of the rectocolored interface, the substrate layer, and the versocolored interface. Once the incident light has crossed the recto-colored interface, it penetrates the substrate where it undergoes multiple scattering. Light scattering in a turbid media is modeled according to different theories depending on the scale on which the interaction of light with the scattering medium is considered. Models based on the radiative transfer equation describe scattering at a microscopic scale. ${ }^{20}$ Such models are computationally heavy and their application to complex media such as paper bulk is not easy. In the case of infinitely large and thin layers of strongly scattering media, ${ }^{21}$ the radiative transfer theory can be reduced to a two-flux model, known as the Kubelka-Munk model. ${ }^{1,7}$ An alternative approach, inspired by the Williams-Clapper model, ${ }^{6}$ considers the interaction of light with the substrate in terms of multiple reflections between the substrate and the print-air interface. In this paper we pursue this approach for modeling the reflectance and transmittance of recto-verso prints.

Reflectance and transmittance of prints depend on the illuminating and measuring conditions, i.e., of the angular distribution of the incident light and of the light captured by the measuring device. This is due to the angular dependence of the attenuations undergone by light when crossing the interfaces and the inked layers, therefore when crossing the colored interfaces. In our model, we separate the expressions of reflectance and transmittance in three components, one depending on the incident light angular distribution, the second depending on the captured light angular distribution, and the third one independent on the illuminating and capturing conditions. The component $T_{\text {in }}$, relative to the penetration of the incident light into the print, represents the fraction of the source flux that reaches the substrate (Fig. 1). It accounts for the attenuations undergone while crossing the rectocolored interface. It depends on the angular distribution of the incident light. The component $T_{\mathrm{ex}}$, relative to the emergence of the detected light, represents the fraction of

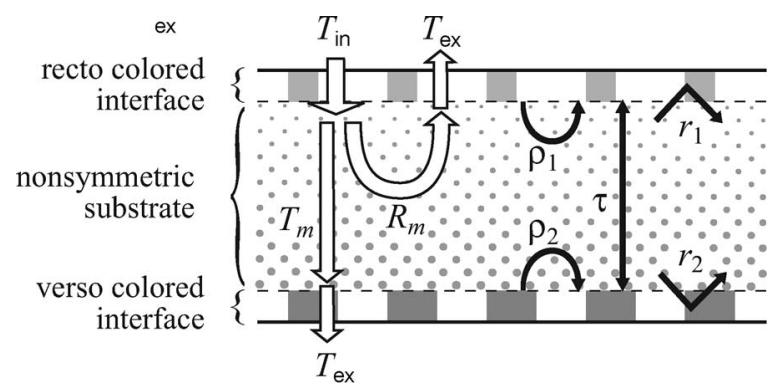

Fig. 1. Interaction of light with the recto-verso print comprising the attenuation of the penetrating light $\left(T_{\text {in }}\right)$, the attenuation of the emerging light $\left(T_{\mathrm{ex}}\right)$, the print's internal reflectance $\left(R_{m}\right)$, and internal transmittance $\left(T_{m}\right)$. The substrate is characterized by its intrinsic reflectance $\rho_{1}$ at its recto side, $\rho_{2}$ at its verso side, and by its intrinsic transmittance $\tau$. The reflectances of the colored interfaces are $r_{1}$ at the recto and $r_{2}$ at the verso. 
total diffuse light emitted by the substrate that reaches the detector. It accounts for the attenuations undergone by the emerging light while crossing the upper (for the print's reflectance) or lower (for the print's transmittance) colored interface. The third component, which is different for the reflectance and the transmittance, comprises the interaction of light inside the print between the substrate and the colored interfaces. The print's internal reflectance $R_{m}$ represents the ratio of the total flux emerging from the substrate at the recto side to the flux received by the substrate from the light source. Likewise, the print's internal transmittance $T_{m}$ represents the ratio of the total flux emerging from the substrate at the verso side to the flux received by the substrate from the light source. Hence, our recto-verso prediction model expresses the global reflectance $R$ of the halftone recto-verso print as

$$
R=T_{\text {in }} T_{\mathrm{ex}} R_{m}
$$

and its global transmittance $T$ as

$$
T=T_{\mathrm{in}} T_{\mathrm{ex}} T_{m} .
$$

We introduce a multiple reflection-transmission model allowing us to express the print's internal reflectance $R_{m}$ and internal transmittance $T_{m}$ by taking into account all possible combinations of successive reflections and transmissions. The internal reflectance and internal transmittance are functions of the recto intrinsic reflectance, the verso intrinsic reflectance and the intrinsic transmittance of the substrate, and the reflectances of the recto-colored interface and of the verso-colored interface.

Before presenting in detail the multiple reflectiontransmission model, we first recall in Section 2 the basic notions of geometric optics necessary for describing the reflection and the transmission of light by an interface. In Section 3 , we present the attenuation $T_{\text {in }}$ of incident light penetrating the print with respect to the illumination geometry and the attenuation $T_{\mathrm{ex}}$ of light exiting the print with respect to the capturing device geometry. After having expressed the print's internal reflectance $R_{m}$ and its internal transmittance $T_{\text {in }}$ (Section 4), we develop the complete expressions of the print's global reflectance and transmittance when the incident light is collimated and the capturing device is a radiance detector (Section 5). We also show how to obtain the corresponding expressions when incident light is diffuse and/or when the exiting light is captured by an integrating sphere. In Section 6, we express both the internal and the intrinsic reflectances and transmittances of the substrate layer as functions of the Kubelka-Munk parameters. For this purpose, we extend the Kubelka-Munk model to recto-verso halftone prints by considering the reflections of light at the colored interfaces as boundary conditions of the Kubelka-Munk differential equation system. We also show that the same expressions for the internal reflectance and transmittance are obtained by inserting into our multiple reflection-transmission model the substrate's intrinsic reflectance and transmittance expressed according to the Kubelka-Munk model. In Section 7, we show that in the case of a solid colorant print, the Williams-Clapper model $^{6}$ is a special case of our multiple reflectiontransmission model. In the case of a halftone print, the classical Clapper-Yule model ${ }^{5}$ also becomes a special case of our model. A method for calibrating the recto-verso reflectance and transmittance model is proposed in Section 8. An experimental verification of the model is performed in Section 9. We draw our conclusions in Section 10.

\section{OPTICS OF AN INTERFACE: BASIC NOTIONS}

The Fresnel reflections and transmissions of light at the print-air interface are of primary importance in a model describing the interaction of light with a print. Different expressions describe the reflection and transmission depending on whether the incident light is collimated or diffuse. We consider the Fresnel reflection and transmission of a collimated irradiance or radiance as well as of a diffuse Lambertian irradiance. The developed expressions are reused in the following sections for colored print-air interfaces.

\section{A. Reflection and Transmission of Collimated Irradiance}

A collimated light flux $\Phi_{i}$ flowing within an infinitesimal solid angle $\mathrm{d} \Omega_{0}$ illuminates an element $\mathrm{d} s$ of a flat interface between a medium $m_{0}$ of refractive index $n_{0}$ and a medium $m_{1}$ of refractive index $n_{1}$. In medium $m_{0}$, the light flux propagates along a direction $\left(\theta_{0}, \phi_{0}\right)$, where $\theta_{0}$ is the polar angle formed with the interface's normal vector, and $\phi_{0}$ is the azimuth angle formed with an arbitrary reference vector of the interface. At the interface, the flux $\Phi_{i}$ is decomposed into a reflected flux $\Phi_{r}=R_{01}\left(\theta_{0}\right) \Phi_{i}$ and a transmitted flux $\Phi_{t}=T_{01}\left(\theta_{0}\right) \Phi_{i}$, where $R_{01}\left(\theta_{0}\right)$ and $T_{01}\left(\theta_{0}\right)$ are the Fresnel reflection and transmission coefficients, given by the Fresnel formulas as functions of the incidence polar angle $\theta_{0}$ and the refractive indices $n_{0}$ and $n_{1}{ }^{22}$

The respective incident, reflected, and transmitted fluxes form with respect to the element of interface $\mathrm{d} s$ an incident irradiance $E_{i}$, a reflected irradiance $E_{r}$, and a transmitted irradiance $E_{t}$. The reflectance of the interface is $E_{r} / E_{i}=R_{01}\left(\theta_{0}\right)$ and its transmittance is $E_{t} / E_{i}=T_{01}\left(\theta_{0}\right)$.

Considering the incident, the reflected, and the transmitted fluxes relatively to their respective propagation directions $\left(\theta_{0}, \phi_{0}\right),\left(\theta_{r}, \phi_{r}\right)$, and $\left(\theta_{1}, \phi_{1}\right)$, their respective infinitesimal solid angles $\mathrm{d} \Omega_{0}=\sin \theta_{0} \mathrm{~d} \theta_{0} \mathrm{~d} \phi_{0}, \quad \mathrm{~d} \Omega_{r}$ $=\sin \theta_{r} \mathrm{~d} \theta_{r} \mathrm{~d} \phi_{r}$, and $\mathrm{d} \Omega_{1}=\sin \theta_{1} \mathrm{~d} \theta_{1} \mathrm{~d} \phi_{1}$, and the interface element $\mathrm{d} s$, we obtain an incident radiance $L_{i}\left(\theta_{0}, \phi_{0}\right)$, a reflected radiance $L_{r}\left(\theta_{0}, \phi_{0}\right)$, and a transmitted radiance $L_{t}\left(\theta_{0}, \phi_{0}\right)$. The incident radiance is defined $\mathrm{as}^{23}$

$$
L_{i}\left(\theta_{0}, \phi_{0}\right)=\frac{\Phi_{i}}{\mathrm{~d} s \cos \theta_{0} \sin \theta_{0} \mathrm{~d} \theta_{0} \mathrm{~d} \phi_{0}} .
$$

According to Snell's laws, the azimuth angles of the reflected and transmitted radiances are $\phi_{r}=\phi_{1}=\phi_{0}+\pi$. The polar angle of the reflected radiance is $\theta_{r}=\theta_{0}$. The polar angle $\theta_{1}$ of the transmitted radiance is related to the angle $\theta_{0}$ by Snell's refraction law:

$$
n_{0} \sin \theta_{0}=n_{1} \sin \theta_{1}
$$

The reflected radiance is 


$$
L_{r}\left(\theta_{0}, \phi_{0}\right)=\frac{R_{01}\left(\theta_{0}\right) \Phi_{i}}{\mathrm{~d} s \cos \theta_{0} \sin \theta_{0} \mathrm{~d} \theta_{0} \mathrm{~d} \phi_{0}}=R_{01}\left(\theta_{0}\right) L_{i}\left(\theta_{0}, \phi_{0}\right)
$$

The product at the left and at the right of Eq. (4) with the differential of Eq. (4) yields

$$
n_{0}^{2} \cos \theta_{0} \sin \theta_{0} \mathrm{~d} \theta_{0}=n_{1}^{2} \cos \theta_{1} \sin \theta_{1} \mathrm{~d} \theta_{1} .
$$

Thanks to Eq. (6) and with $\mathrm{d} \phi_{0}=\mathrm{d} \phi_{1}$, the transmitted radiance, initially defined as

$$
L_{t}\left(\theta_{0}, \phi_{0}\right)=\frac{T_{01}\left(\theta_{0}\right) \Phi_{i}}{\mathrm{~d} s \cos \theta_{1} \sin \theta_{1} \mathrm{~d} \theta_{1} \mathrm{~d} \phi_{1}},
$$

can also be expressed as

$$
L_{t}\left(\theta_{0}, \phi_{0}\right)=\left(n_{1} / n_{0}\right)^{2} T_{01}\left(\theta_{0}\right) L_{i}\left(\theta_{0}, \phi_{0}\right) .
$$

The term $\left(n_{1} / n_{0}\right)^{2}$ is characteristic of the transmission of radiance across an interface from medium $m_{0}$ to medium $m_{1}$ and accounts for the change in solid angle and projected area due to the refraction.

\section{B. Properties of the Fresnel Reflection and Transmission Coefficients}

Since the energy is conserved at the interface, the Fresnel reflection and transmission coefficients $R_{01}\left(\theta_{0}\right)$ and $T_{01}\left(\theta_{0}\right)$ satisfy the relationship

$$
R_{01}\left(\theta_{0}\right)+T_{01}\left(\theta_{0}\right)=1 .
$$

We have the same Fresnel transmission coefficient both for an incident flux from medium $m_{0}$ with an angle $\theta_{0}$ and for an incident flux from medium $m_{1}$ with an angle $\theta_{1}$ related to angle $\theta_{0}$ by Eq. (4):

$$
T_{01}\left(\theta_{0}\right)=T_{10}\left(\theta_{1}\right) .
$$

The combination of Eqs. (8) and (9) yields

$$
\begin{gathered}
R_{01}\left(\theta_{0}\right)=R_{10}\left(\theta_{1}\right), \\
R_{10}\left(\theta_{1}\right)+T_{10}\left(\theta_{1}\right)=1 .
\end{gathered}
$$

\section{Reflection and Transmission of Lambertian Irradiance by an Interface}

Let us now consider that the interface is illuminated by Lambertian irradiance incident from medium $m_{0}$. A proportion $r_{01}$, called diffuse reflectance of the interface, is reflected by the interface. A proportion $t_{01}$, called diffuse transmittance, is transmitted across the interface. If the interface is illuminated by a Lambertian irradiance from medium $m_{1}$, the diffuse reflectance and diffuse transmittance are called $r_{10}$ and $t_{10}$. We present in detail the calculation of $r_{01}$. The other expressions, $t_{01}, r_{10}$, and $t_{10}$, can be obtained by following the same line of reasoning.

Let us call $E_{i}$ the incident Lambertian irradiance. The interface receives from each direction $\left(\theta_{0}, \phi_{0}\right)$ of the hemisphere the same radiance $L_{i}=E_{i} / \pi$, contained within the infinitesimal solid angle $\mathrm{d} \Omega_{0}=\sin \theta_{0} \mathrm{~d} \theta_{0} \mathrm{~d} \phi_{0}$. The corresponding element of irradiance $\mathrm{d} E_{i}\left(\theta_{0}, \phi_{0}\right)$ is

$$
\mathrm{d} E_{i}\left(\theta_{0}, \phi_{0}\right)=L_{i} \cos \theta_{0} \mathrm{~d} \Omega_{0}=\frac{E_{i}}{\pi} \cos \theta_{0} \sin \theta_{0} \mathrm{~d} \theta_{0} \mathrm{~d} \phi_{0} .
$$

This element of irradiance $\mathrm{d} E_{i}\left(\theta_{0}, \phi_{0}\right)$ is reflected by the interface in a proportion $R_{01}\left(\theta_{0}\right)$ given by the Fresnel formulas. The reflected element of irradiance $\mathrm{d} E_{r}\left(\theta_{0}, \phi_{0}\right)$ is therefore

$$
\mathrm{d} E_{r}\left(\theta_{0}, \phi_{0}\right)=R_{01}\left(\theta_{0}\right) \frac{E_{i}}{\pi} \cos \theta_{0} \sin \theta_{0} \mathrm{~d} \theta_{0} \mathrm{~d} \phi_{0} .
$$

The total reflected irradiance $E_{r}$ is the sum over the hemisphere of the reflected elements of irradiance:

$$
E_{r}=\int_{\phi_{0}=0}^{2 \pi} \int_{\theta_{0}=0}^{\pi / 2} R_{01}\left(\theta_{0}\right) \frac{E_{i}}{\pi} \cos \theta_{0} \sin \theta_{0} \mathrm{~d} \theta_{0} \mathrm{~d} \phi_{0} .
$$

Since the integrated terms do not depend on $\phi_{0}$, the integration according to $\phi_{0}$ yields a factor of $2 \pi$. After rearranging, Eq. (13) becomes

$$
E_{r}=E_{i} \int_{\theta_{0}=0}^{\pi / 2} R_{01}\left(\theta_{0}\right) \sin 2 \theta_{0} \mathrm{~d} \theta_{0} \mathrm{~d} \phi_{0} .
$$

The external diffuse reflectance $r_{01}$ of the interface, given by the ratio $E_{r} / E_{i}$, was first presented by $\mathrm{Judd}^{24}$ :

$$
r_{01}=\int_{\theta_{0}=0}^{\pi / 2} R_{01}\left(\theta_{0}\right) \sin 2 \theta_{0} \mathrm{~d} \theta_{0}
$$

Similarly, the external diffuse transmittance of the interface $t_{01}$ is

$$
t_{01}=\int_{\theta_{0}=0}^{\pi / 2} T_{01}\left(\theta_{0}\right) \sin 2 \theta_{0} \mathrm{~d} \theta_{0} .
$$

Equation (8) expresses the conservation of energy at the interface. By inserting this relation into the integral of Eq. (16), we may verify that the energy is also conserved for a diffuse illumination:

$$
t_{01}=1-r_{01} \text {. }
$$

The diffuse reflectance of the interface $r_{10}$ is

$$
r_{10}=\int_{\theta_{1}=0}^{\pi / 2} R_{10}\left(\theta_{1}\right) \sin 2 \theta_{1} \mathrm{~d} \theta_{1},
$$

and the diffuse transmittance of the interface $t_{10}$ is

$$
t_{10}=\int_{\theta_{1}=0}^{\pi / 2} T_{10}\left(\theta_{1}\right) \sin 2 \theta_{1} \mathrm{~d} \theta_{1}=1-r_{10} .
$$

Let us establish the relationship between $t_{01}$ [Eq. (16)] and $t_{10}$ [Eq. (19)]. According to Eq. (9), $T_{01}\left(\theta_{0}\right)=T_{10}\left(\theta_{1}\right)$, and according to Eq. (6), $n_{0}^{2} \sin 2 \theta_{0} \mathrm{~d} \theta_{0}=n_{1}^{2} \sin 2 \theta_{1} \mathrm{~d} \theta_{1}$. Thanks to these equalities, we obtain the relation

$$
t_{10}=\left(n_{0} / n_{1}\right)^{2} t_{01} \text {. }
$$

Numerical values of $r_{01}, t_{01}, r_{10}$, and $t_{10}$ are given in Appendix A for a relative index of refraction of 1.5. Once the numerical value of $r_{01}$ is known, the values of $t_{01}, r_{10}$, and $t_{10}$ can be directly deduced from Eqs. (17), (19), and (20). 


\section{Reflection of Lambertian Irradiance by a Colored Interface}

A colored interface, i.e., an interface adjacent to an inked layer, illuminated by Lambertian irradiance at the side of the inked layer has a reflectance $r(t)$ depending on the inked layer transmittance $t$. Since the inked layer is nonscattering, light rays cross the inked layer along straight lines having a path length and therefore an attenuation that depend on their orientation. Thus, the diffuse reflectance of colored interfaces accounts for the attenuation within the inked layer and for the Fresnel reflection at the interface for each direction of propagation of the incident light.

The Lambertian irradiance $E_{i}$, coming from medium $m_{1}$, is decomposed into elements of irradiance $\mathrm{d} E_{i}\left(\theta_{1}, \phi_{1}\right)$ [Eq. (12)]. Each element of irradiance is reflected by the interface with a factor of $R_{10}\left(\theta_{1}\right)$ and traverses twice, before and after the reflection, a path of relative length $1 / \cos \theta_{1}$ within the inked layer. According to Beer's law, the element of irradiance is attenuated by a factor $t^{2 / \cos \theta_{1}}$, with $t$ being the attenuation undergone by the light crossing the inked layer along its normal direction. The reflected element of irradiance is

$$
\mathrm{d} E_{r}\left(\theta_{1}, \phi_{1}\right)=t^{2 / \cos \theta_{1}} R_{10}\left(\theta_{1}\right) \frac{E_{i}}{\pi} \cos \theta_{1} \sin \theta_{1} \mathrm{~d} \theta_{1} \mathrm{~d} \phi_{1} .
$$

We sum up all the reflected elements of irradiance, yielding the reflected irradiance $E_{r}$, and carry out the same simplifications as for Eq. (14). The resulting expression for the reflectance of the colored interface, given by the ratio $E_{r} / E_{i}$, has been first introduced by Williams and Clapper $^{6}$ :

$$
r(t)=\int_{\theta_{1}=0}^{\pi / 2} t^{2 / \cos \theta_{1}} R_{10}\left(\theta_{1}\right) \sin 2 \theta_{1} \mathrm{~d} \theta_{1} .
$$

In Table 2 (see Appendix A), for a print-air relative refractive index of 1.5, we tabulate numerical values of $r(t)$ as a function of the colorant layer normal transmittance $t$.

In halftone patches, the colored interface is composed of several colorant areas of respective transmittances $t_{k}$ and surface coverages $a_{k}$. In each colorant area, the internal reflectance is $r\left(t_{k}\right)$. Each colorant area contributes to the total reflectance in proportion to its surface coverage $a_{k}$. Therefore,

$$
r(t)=\sum_{k} a_{k} r\left(t_{k}\right)=\sum_{k} a_{k} \int_{\theta_{1}=0}^{\pi / 2} t_{k}^{2 / \cos \theta_{1}} R_{10}\left(\theta_{1}\right) \sin 2 \theta_{1} \mathrm{~d} \theta_{1} .
$$

In Eq. (23), we ignore the fact that some oblique light rays, located close to the frontier between two colorants, may start their path in a colorant and finish it in a different colorant. This phenomenon concerns mainly light rays oriented according to a large angle. However, since the term $t_{k}^{2 / \cos \theta_{1}}$ decreases rapidly toward zero as angle $\theta_{1}$ approaches $\pi / 2$, and since the colorant areas are large compared with the inked layer's thickness, we assume that the contribution of highly inclined light rays internally reflected at the frontier between two colorant areas is negligible.

\section{PENETRATION AND EMERGENCE OF THE LIGHT ACROSS THE COLORED INTERFACES}

The incident light is attenuated by a factor $T_{\text {in }}$ during its path from the air (medium $m_{0}$ of refractive index $n_{0}=1$ denoted by subscript 0 ) to the substrate (medium $m_{1}$ of refractive index $n_{1}$ denoted by the subscript 1 ). We call $n=n_{1} / n_{0}$ the relative refractive index of the interface between media $m_{0}$ and $m_{1}$. The factor $T_{\text {in }}$ depends on the geometry of illumination. We therefore develop an expression $T_{\text {in }}\left(\theta_{0}\right)$ for a collimated illumination at incidence $\theta_{0}$ and an expression $T_{\text {in }}^{(d)}$ for a diffuse illumination.

Let us first consider a collimated incident light forming an angle $\theta_{0}$ with the normal of the print. A fraction $R_{01}\left(\theta_{0}\right)$ is specularly reflected at the air side of the recto interface. We ignore this specular reflection since, in most measuring instruments, it can be discarded from the measurements. The rest of the light, i.e., a fraction $T_{01}\left(\theta_{0}\right)$, is transmitted across the recto interface with an angle $\theta_{1}$ according to Snell's law [Eq. (4)]. It crosses the ink layer of normal transmittance $t$ along a path of relative length

$$
\mu\left(\theta_{0}\right)=1 / \cos \theta_{1}=\left[1-\left(\sin \theta_{0} / n\right)^{2}\right]^{-1 / 2}
$$

and therefore undergoes, according to Beer's law, ${ }^{25}$ an attenuation $t^{\mu\left(\theta_{0}\right)}$ within the inked layer. It then penetrates the diffusing substrate. The total attenuation factor $T_{\text {in }}\left(\theta_{0}\right)$ applied to the incident irradiance oriented with an angle $\theta_{0}$ while passing from the air to the diffusing substrate is

$$
T_{\text {in }}\left(\theta_{0}\right)=T_{01}\left(\theta_{0}\right) t^{\mu\left(\theta_{0}\right)} .
$$

If the inked layer is composed of several colorants of respective transmittances $t_{k}$ and fractional surface coverages $a_{k}$, the attenuation factor $T_{\text {in }}\left(\theta_{0}\right)$ becomes

$$
T_{\text {in }}\left(\theta_{0}\right)=T_{01}\left(\theta_{0}\right) \sum_{k} a_{k} t_{k}^{\mu\left(\theta_{0}\right)}
$$

We now consider Lambertian illumination. Let us follow the same line of reasoning as for the case of an interface, presented in Section 2 [Eqs. (12)-(15)]. Instead of considering the reflection of the elements of irradiance $\mathrm{d} E_{i}\left(\theta_{0}, \phi_{0}\right)$ with a proportion $R_{01}\left(\theta_{0}\right)$, we consider their transmission across the interface with a proportion $T_{01}\left(\theta_{0}\right)$ and across the inked layer with a proportion $t^{\mu\left(\theta_{0}\right)}$. Thus, the fraction $T_{\text {in }}^{(d)}$ of incident irradiance that is transmitted across the colored interface has the same expression as in Eq. (15), where $R_{01}\left(\theta_{0}\right)$ is replaced by $T_{01}\left(\theta_{0}\right) t^{\mu\left(\theta_{0}\right)}$ :

$$
T_{\text {in }}^{(d)}=\int_{\theta_{0}=0}^{\pi / 2} T_{01}\left(\theta_{0}\right) t^{\mu\left(\theta_{0}\right)} \sin 2 \theta_{0} \mathrm{~d} \theta_{0} .
$$

Equation (27) can be approximated with excellent accuracy by separating the transmission across the interface and the transmission across the inked layer. The incident Lambertian irradiance $E_{i}$ is first transmitted across the 
interface according to a proportion $t_{01}$ [Eq. (16)]. The irradiance $t_{01} E_{i}$ crosses the inked layer and is attenuated by a factor $t^{\mu}$. The total attenuation applied to the incident irradiance is therefore approximated as

$$
T_{\text {in }}^{(d)} \approx t^{\mu} t_{01} .
$$

The exponent $\mu$ is obtained numerically, for a given refractive index $n$, by minimizing the sum of the squared differences between approximation (28) and the exact expression given in Eq. (27) (see Appendix B). For $n=1.5$, the optimal value of $\mu$ is 1.13 . The difference between the exact and the approximated values of $T_{\text {in }}$ is inferior to 0.001 . Intuitively, $\mu$ represents an average path traversed by the light in the inked layer.

When the inked layer is composed of several colorants, of respective transmittances $t_{k}$ and surface coverages $a_{k}$, the approximated attenuation factor $T_{\text {in }}^{(d)}$ becomes

$$
T_{\mathrm{in}}^{(d)}=t_{01} \sum_{k} a_{k} t_{k}^{\mu}
$$

We proceed for the emerging light in the same manner as for the penetration of the incident light, i.e., we express the factor $T_{\text {ex }}$ relative to the light path from the substrate to the detector, according to the geometry of observation. We develop an expression $T_{\text {ex }}\left(\theta_{0}^{\prime}\right)$ for the capture of light by a radiance detector at an angle $\theta_{0}$ and an expression $T_{\mathrm{ex}}^{(d)}$ for the capture of light by a integrating sphere.

Let us first consider that the measuring device is a radiance detector, which captures the light exiting the print with an angle $\theta_{0}^{\prime}$. In the print, the substrate emits a Lambertian irradiance $E_{p}$ toward the interface, i.e., a constant radiance $E_{p} / \pi$ in all directions of the hemisphere. Only the radiance emitted into the particular direction $\theta_{1}^{\prime}$, such that $n \sin \theta_{1}^{\prime}=\sin \theta_{0}^{\prime}$, is captured by the radiance detector. It first crosses the inked layer along a path of relative length $1 / \cos \theta_{1}^{\prime}=\mu\left(\theta_{0}^{\prime}\right)$, expressed as in Eq. (24), and is attenuated by $t^{\mu\left(\theta_{0}^{\prime}\right)}$. Then the radiance $t^{\mu\left(\theta_{0}^{\prime}\right)} E_{p} / \pi$ crosses the interface. According to Eq. (7) and the fact that this radiance is transmitted from medium $m_{1}$ to medium $m_{0}$, the transmitted radiance $L_{d}$ that the detector captures is

$$
L_{d}=(1 / n)^{2} T_{10}\left(\theta_{1}^{\prime}\right) t^{\mu\left(\theta_{0}^{\prime}\right)} E_{p} / \pi,
$$

where $T_{10}\left(\theta_{1}^{\prime}\right)=T_{01}\left(\theta_{0}^{\prime}\right)$ according to Eq. (9). Thus, the fraction $T_{\text {ex }}\left(\theta_{0}^{\prime}\right)$ of the irradiance $E_{p}$ emitted by the substrate and captured by the radiance detector at angle $\theta_{0}^{\prime}$ is

$$
T_{\mathrm{ex}}\left(\theta_{0}^{\prime}\right)=\frac{L_{d}}{E_{p}}=\frac{T_{01}\left(\theta_{0}^{\prime}\right)}{\pi n^{2}} t^{\mu\left(\theta_{0}^{\prime}\right)},
$$

or, for an inked layer composed of several colorants,

$$
T_{\text {ex }}\left(\theta_{0}^{\prime}\right)=\frac{T_{01}\left(\theta_{0}^{\prime}\right)}{\pi n^{2}} \sum_{k} a_{k} t_{k}^{\mu\left(\theta_{0}^{\prime}\right)} .
$$

The integrating sphere captures the total emerging irradiance, which corresponds to a fraction $T_{\mathrm{ex}}^{(d)}$ of the irradiance $E_{p}$ emitted by the substrate. Let us decompose the incident irradiance $E_{p}$ into elements of irradiance $\mathrm{d} E\left(\theta_{1}^{\prime}, \phi_{1}^{\prime}\right)$. We express their attenuation as a function of their orientation $\left(\theta_{1}^{\prime}, \phi_{1}^{\prime}\right)$ and sum them up to obtain the total emerging irradiance. Since the irradiance $E_{p}$ emitted by the substrate is Lambertian, the elements of irradiance $\mathrm{d} E\left(\theta_{1}^{\prime}, \phi_{1}^{\prime}\right)$ are

$$
\mathrm{d} E\left(\theta_{1}^{\prime}, \phi_{1}^{\prime}\right)=\frac{E_{p}}{\pi} \cos \theta_{1}^{\prime} \sin \theta_{1}^{\prime} \mathrm{d} \theta_{1}^{\prime} \mathrm{d} \phi_{1}^{\prime} .
$$

They undergo an attenuation $t^{1 / \cos \theta_{1}^{\prime}}$ in the ink layer and are transmitted into the air by a factor of $T_{10}\left(\theta_{1}^{\prime}\right)$. We sum up all the emerging elements of irradiance whose incident direction $\left(\theta_{1}^{\prime}, \phi_{1}^{\prime}\right)$ belongs to the hemisphere. The resulting integral yields the emerging irradiance $E$. As previously, the integration according to the azimuth angle yields a factor of $2 \pi$, and the expression $2 \cos \theta_{1}^{\prime} \sin \theta_{1}^{\prime}$ is replaced with $\sin 2 \theta_{1}^{\prime}$. The emerging irradiance is therefore

$$
E=E_{p} \int_{\theta_{1}^{\prime}=0}^{\pi / 2} t^{1 / \cos \theta_{1}^{\prime}} T_{10}\left(\theta_{1}^{\prime}\right) \sin 2 \theta_{1}^{\prime} \mathrm{d} \theta_{1}^{\prime} .
$$

The ratio $E / E_{p}$ yields the attenuation $T_{\mathrm{ex}}^{(d)}$ of the diffuse light emerging from the substrate into the air:

$$
T_{\mathrm{ex}}^{(d)}=\int_{\theta_{1}^{\prime}=0}^{\pi / 2} t^{1 / \cos \theta_{1}^{\prime}} T_{10}\left(\theta_{1}^{\prime}\right) \sin 2 \theta_{1}^{\prime} \mathrm{d} \theta_{1}^{\prime} .
$$

Note that when $\theta_{1}^{\prime}$ exceeds $\arcsin (1 / n)$, the element of irradiance is totally reflected and $T_{10}\left(\theta_{1}^{\prime}\right)$ is zero. The integration domain in Eq. (32) can therefore be reduced to $[0, \arcsin (1 / n)]$. We may also express the terms under the integral as a function of $\theta_{0}^{\prime}$, the angle in the air, instead of $\theta_{1}^{\prime}$, the angle in the print. The term $T_{10}\left(\theta_{1}^{\prime}\right)$ is equal to $T_{01}\left(\theta_{0}^{\prime}\right)$. The term $1 / \cos \theta_{1}^{\prime}$ was defined as $\mu\left(\theta_{0}^{\prime}\right)$ in Eq. (24). According to Eq. (6), $\sin 2 \theta_{1}^{\prime} \mathrm{d} \theta_{1}^{\prime}$ is equal to $(1 / n)^{2} \sin 2 \theta_{0}^{\prime} \mathrm{d} \theta_{0}^{\prime}$. The integration domain according to $\theta_{0}^{\prime}$ is $[0, \pi / 2]$ and Eq. (33) becomes

$$
T_{\mathrm{ex}}^{(d)}=\frac{1}{n^{2}} \int_{\theta_{1}^{\prime}=0}^{\pi / 2} t^{\mu\left(\theta_{0}^{\prime}\right)} T_{01}\left(\theta_{0}^{\prime}\right) \sin 2 \theta_{0}^{\prime} \mathrm{d} \theta_{0}^{\prime} .
$$

The same approximation that was applied to Eq. (27) also applies to Eq. (33), i.e., we consider separately the attenuation $t^{\mu}$ applied to the Lambertian irradiance in the inked layer and its transmission across the interface:

$$
T_{\mathrm{ex}}^{(d)} \approx t^{\mu} t_{10}
$$

where $t_{10}$ is the transmittance of the interface, expressed in Eq. (19). When the inked layer is composed of several colorants, of respective transmittances $t_{k}$ and surface coverages $a_{k}$, the attenuation factor $T_{\mathrm{ex}}^{(d)}$ becomes

$$
T_{\mathrm{ex}}^{(d)} \approx t_{10} \sum_{k} a_{k} t_{k}^{\mu}
$$

Since light can exit the print across both interfaces, we have one factor $T_{\mathrm{ex}}$ for light exiting at the recto, and another factor $T_{\text {ex }}$ for light exiting at the verso. Their expressions are given by approximation (35) or (36), with the surface coverages $a_{k}$ and colorant transmittances $t_{k}$ relative to, respectively, the colorants printed on the recto and on the verso of the print. 


\section{MULTIPLE INTERNAL REFLECTIONS AND TRANSMISSIONS BETWEEN THE PRINT'S INTERFACES}

Once the incident light has penetrated the print, and before it emerges across the recto- or the verso-colored interface, light is scattered multiple times within the substrate (see Fig. 1). At the recto, light may be reflected by the recto-colored interface (reflectance $r_{1}$ ) or by the substrate (intrinsic reflectance $\rho_{1}$ ). At the verso, light may be reflected by the verso-colored interface (reflectance $r_{2}$ ) or by the substrate (intrinsic reflectance $\rho_{2}$ ). Light may also be transmitted through the substrate (intrinsic transmittance $\tau$ ). Considering the multiple reflections and transmissions occurring within the substrate layer bounded by the colored interfaces, the fraction of penetrating light ready to emerge at the recto is $R_{m}$, the internal reflectance of the print, and the fraction ready to emerge at the verso is $T_{m}$, the internal transmittance of the print.

The multiple paths followed by light before exiting the print are illustrated by the infinite graph of Fig. 2, where arcs represent interactions of the light with the substrate (reflection or transmission by the substrate layer) and nodes represent interactions with the colored interfaces (internal reflection). At the starting node we have the incident irradiance $W_{i}$, which has already crossed the rectocolored interface. All other nodes of the graph are possible ending nodes, where light may exit the print. Between the starting node and a given ending node, light may follow any of the possible paths along the directed arcs of the graph. Along a given path, an attenuation factor is applied at each encountered arc and node. Horizontal arcs, weighted by a factor $\rho_{1}$ or $\rho_{2}$, represent a reflection by the substrate, respectively, at the recto or verso side. Vertical arcs, weighted by a factor $\tau$, represent a transmission across the substrate. Nodes circled with a light line, weighted by a factor $r_{1}$, represent an internal reflection at the recto-colored interface. Nodes circled with a bold line, weighted by a factor $r_{2}$, represent an internal reflection at the verso-colored interface. Horizontal arcs link two nodes relative to a same interface and vertical arcs link two nodes relative to different interfaces. Thus, in the graph of Fig. 2, the nodes belonging to the same horizontal line are relative to the same (recto- or verso-) colored interface.

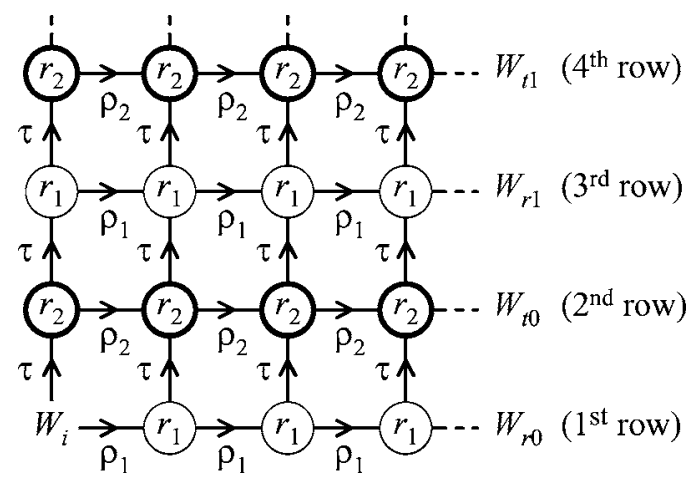

Fig. 2. Representation of the paths followed by the diffuse light within the print, accounting for the reflections by the substrate (reflectance $\rho_{1}$ at the recto side and $\rho_{2}$ at the verso side), the transmissions by the substrate (transmittance $\tau$ ), and the reflections at the colored interfaces (reflectance $r_{1}$ at the recto and $r_{2}$ at the verso).
Let us calculate the total internal irradiance $W_{r}$ that is ready to emerge at the recto side, which contributes to the print's reflectance, and the total internal irradiance $W_{t}$ that is ready to emerge at the verso side, which contributes to the print's transmittance.

We call $W_{r 0}$ the sum of the irradiances corresponding to the paths ending at the nodes of the first row:

$$
W_{r 0}=W_{i}\left[\rho_{1}+r_{1} \rho_{1}^{2}+r_{1}^{2} \rho_{1}^{3}+\ldots\right]=W_{i} \rho_{1}\left[1+r_{1} \rho_{1}+r_{1}^{2} \rho_{1}^{2} \ldots\right] .
$$

Equation (37) contains a geometric series converging toward $1 /\left(1-r_{1} \rho_{1}\right)$. The irradiance $W_{r 0}$ contributes to the total illumination of the recto-colored interface:

$$
W_{r 0}=W_{i} \frac{\rho_{1}}{1-r_{1} \rho_{1}} .
$$

We call $W_{r 0}$ the sum of the irradiances corresponding to the paths ending at the nodes of the second row. Such paths follow exactly one vertical arc. Let us first consider the paths following the first vertical arc (leftmost vertical arc starting at $W_{i}$ ). Once having followed the vertical arc (attenuation factor $\tau$ ), they may continue along the second row, each following arc yielding an attenuation factor $\rho_{2}$ and each crossed node yielding an attenuation factor $r_{2}$. In analogy with Eqs. (37) and (38), the sum of the corresponding irradiances yields a geometrical series $1 /(1$ $\left.-r_{2} \rho_{2}\right)$. Therefore, the irradiance corresponding to the paths following the first vertical arc and ending at the nodes of the second row is $W_{i} \tau /\left(1-r_{2} \rho_{2}\right)$.

We now consider the paths following the second vertical arc. Prior to following the vertical arc, they follow one horizontal arc (attenuation factor $\rho_{1}$ ) and cross one node on the first row (attenuation factor $r_{1}$ ). They follow the vertical arc (attenuation factor $\tau$ ) and then may continue along the second row [yielding a geometrical series $1 /(1$ $\left.\left.-r_{2} \rho_{2}\right)\right]$. These paths contribute to an irradiance $W_{i} \rho_{1} r_{1} \tau /\left(1-r_{2} \rho_{2}\right)$.

By considering all the successive vertical arcs, and by summing all the resulting irradiances, we obtain for the second row a total irradiance $W_{t 0}$, contributing to the total illumination of the verso-colored interface:

$$
W_{t 0}=W_{i}\left[\frac{\tau}{1-r_{2} \rho_{2}}+r_{1} \rho_{1} \frac{\tau}{1-r_{2} \rho_{2}}+\left(r_{1} \rho_{1}\right)^{2} \frac{\tau}{1-r_{2} \rho_{2}}+\ldots\right]
$$

After factorizing Eq. (39) by the term $\tau /\left(1-r_{2} \rho_{2}\right)$, the infinite sum yields a geometric series converging toward $1 /\left(1-r_{1} \rho_{1}\right)$. The expression of $W_{t 0}$ thus becomes

$$
W_{t 0}=W_{i} \frac{\tau}{\left(1-r_{1} \rho_{1}\right)\left(1-r_{2} \rho_{2}\right)} .
$$

We call $W_{r 1}$ the sum of the irradiances corresponding to the paths ending at the nodes of the third row. This irradiance can be derived from the irradiance $W_{t 0}$ by considering that, instead of ending at the nodes of the second row, the paths cross one additional node on the second row (attenuation factor $r_{2}$ ), follow one vertical arc (attenuation factor $\tau$ ), and continue along the third row [geometrical series $\left.1 /\left(1-r_{1} \rho_{1}\right)\right]$. We obtain for the third row a 
total irradiance $W_{r 1}$, contributing to the total illumination of the recto-colored interface:

$$
W_{r 1}=W_{t 0} \frac{r_{2} \tau}{\left(1-r_{1} \rho_{1}\right)}=W_{i} \frac{r_{2} \tau^{2}}{\left(1-r_{1} \rho_{1}\right)^{2}\left(1-r_{2} \rho_{2}\right)} .
$$

We call $W_{t 1}$ the sum of the irradiances corresponding to the paths ending at the nodes of the fourth row. As above, the irradiance $W_{t 1}$ can be derived from the irradiance $W_{r 1}$ by considering that, instead of ending at the nodes of the third row, the paths cross one additional node on the third row (attenuation factor $r_{1}$ ), follow one vertical arc (attenuation factor $\tau$ ), and continue along the fourth row [geometrical series $\left.1 /\left(1-r_{2} \rho_{2}\right)\right]$. We obtain for the fourth row a total irradiance $W_{t 1}$, contributing to the total illumination of the verso-colored interface:

$$
W_{t 1}=W_{i} \frac{r_{1} r_{2} \tau^{3}}{\left(1-r_{1} \rho_{1}\right)^{2}\left(1-r_{2} \rho_{2}\right)^{2}} .
$$

By considering any of the successive rows, we obtain the generic expression of the irradiances $W_{r k}$ contributing to the illumination of the recto-colored interface,

$$
W_{r k}=W_{i} \frac{r_{1}^{k-1} r_{2}^{k} \tau^{2 k}}{\left(1-r_{1} \rho_{1}\right)^{k+1}\left(1-r_{2} \rho_{2}\right)^{k}}, \quad k=1,2 \ldots
$$

and the generic expression of the irradiances $W_{t k}$ contributing to the illumination of the verso-colored interface,

$$
W_{t k}=W_{i} \frac{r_{1}^{k} r_{2}^{k} \tau^{2 k+1}}{\left(1-r_{1} \rho_{1}\right)^{k+1}\left(1-r_{2} \rho_{2}\right)^{k+1}}, \quad k=0,1,2 \ldots
$$

The total irradiance $W_{r}$ ready to emerge at the recto is given by the sum of the irradiance $W_{r k}, k=0,1,2 \ldots$, yielding a geometric series

$$
\begin{aligned}
W_{r}= & W_{i} \frac{\rho_{1}}{1-r_{1} \rho_{1}}+W_{i} \frac{1}{r_{1}\left(1-r_{1} \rho_{1}\right)} \sum_{k=1}^{\infty}\left[\frac{r_{1} r_{2} \tau^{2}}{\left(1-r_{1} \rho_{1}\right)\left(1-r_{2} \rho_{2}\right)}\right]^{k} \\
= & W_{i}\left[\frac{\rho_{1}}{1-r_{1} \rho_{1}}\right. \\
& \left.+\frac{1}{r_{1}\left(1-r_{1} \rho_{1}\right)} \cdot \frac{r_{1} r_{2} \tau^{2}}{\left(1-r_{1} \rho_{1}\right)\left(1-r_{2} \rho_{2}\right)-r_{1} r_{2} \tau^{2}}\right] \\
= & W_{i} \frac{\rho_{1}-r_{2}\left(\rho_{2} \rho_{1}-\tau^{2}\right)}{\left(1-r_{1} \rho_{1}\right)\left(1-r_{2} \rho_{2}\right)-r_{1} r_{2} \tau^{2}} .
\end{aligned}
$$

Similarly, the total irradiance $W_{t}$ ready to emerge at the verso is given by the sum of the irradiance $W_{t k}, k$ $=0,1,2 \ldots$, yielding also a geometric series

$$
\begin{aligned}
W_{t} & =W_{i} \frac{\tau}{\left(1-r_{1} \rho_{1}\right)\left(1-r_{2} \rho_{2}\right)} \sum_{k=0}^{\infty}\left[\frac{r_{1} r_{2} \tau^{2}}{\left(1-r_{1} \rho_{1}\right)\left(1-r_{2} \rho_{2}\right)}\right]^{k} \\
& =W_{i} \frac{\tau}{\left(1-r_{1} \rho_{1}\right)\left(1-r_{2} \rho_{2}\right)-r_{1} r_{2} \tau^{2}} .
\end{aligned}
$$

Finally, the print's internal reflectance $R_{m}$ obtained according to our multiple reflection-transmission model is given by the ratio $W_{r} / W_{i}$,

$$
R_{m}=\frac{\rho_{1}-r_{2}\left(\rho_{1} \rho_{2}-\tau^{2}\right)}{\left(1-r_{1} \rho_{1}\right)\left(1-r_{2} \rho_{2}\right)-r_{1} r_{2} \tau^{2}},
$$

and the internal transmittance $T_{m}$ by the ratio $W_{t} / W_{i}$,

$$
T_{m}=\frac{\tau}{\left(1-r_{1} \rho_{1}\right)\left(1-r_{2} \rho_{2}\right)-r_{1} r_{2} \tau^{2}} .
$$

In the case where the print support is composed of a uniform substrate layer, i.e., $\rho_{1}=\rho_{2}=\rho$, Eqs. (40) and (41) become

$$
\begin{aligned}
& R_{m}=\frac{\rho-r_{2}\left(\rho^{2}-\tau^{2}\right)}{\left(1-r_{1} \rho\right)\left(1-r_{2} \rho\right)-r_{1} r_{2} \tau^{2}}, \\
& T_{m}=\frac{\tau}{\left(1-r_{1} \rho\right)\left(1-r_{2} \rho\right)-r_{1} r_{2} \tau^{2}} .
\end{aligned}
$$

We now have expressions for all the components of our recto-verso prediction model: $T_{\text {in }}$ (Section 3), $T_{\text {ex }}$ (Section 3), $R_{m}$ [Eq. (40)], and $T_{m}$ [Eq. (41)]. We may therefore express the global reflectance and the global transmittance of a recto-verso print.

\section{GLOBAL REFLECTANCE AND TRANSMITTANCE OF A HALFTONE PRINT}

According to the recto-verso model, represented by Eqs. (2) and (3), the global reflectance (transmittance) of a halftone print comprises the terms representing the penetration of incident light, the print's internal reflectance (transmittance), and the term representing the emergence of detected light.

Let us consider, for example, a print with a solid ink of transmittance $t_{1}$ on the recto and a solid ink of transmittance $t_{2}$ on the verso. The internal reflectance of the rectoand the verso-colored interfaces are, respectively, $r_{1}$ $=r\left(t_{1}\right)$ and $r_{2}=r\left(t_{2}\right)$, with function $r$ expressed by Eq. (22). We consider a measuring geometry where collimated light is incident at an angle $\theta_{0}$ and reflected light is captured by a radiance detector at an angle $\theta_{0}^{\prime}$. The term $T_{\text {in }}\left(\theta_{0}\right)$ corresponding to the penetration of the collimated incident light is given by Eq. (25). The term $T_{\text {ex }}\left(\theta_{0}^{\prime}\right)$ corresponding to the emergence of light toward the radiance detector at the recto is given by Eq. (30) with $t=t_{1}$. Another radiance detector captures the light emerging at the verso at an angle $\theta_{0}^{\prime \prime}$. The corresponding term $T_{\text {ex }}\left(\theta_{0}^{\prime \prime}\right)$ is given by Eq. (30) with $t=t_{2}$.

We derive from Eq. (2) a specific expression for the global reflectance $R$ of the print composed of a solid ink on the recto and a solid ink on the verso for a collimated illumination at angle $\theta_{0}$, a radiance detector at angle $\theta_{0}^{\prime}$, and for light penetrating and emerging through the rectocolored interface:

$$
\begin{aligned}
R= & T_{\text {in }}\left(\theta_{0}\right) T_{\mathrm{ex}}\left(\theta_{0}^{\prime}\right) R_{m} \\
= & T_{01}\left(\theta_{0}\right) \frac{T_{01}\left(\theta_{0}^{\prime}\right)}{\pi n^{2}} t_{1}^{\mu\left(\theta_{0}\right)+\mu\left(\theta_{0}^{\prime}\right)} \\
& \times \frac{\rho_{1}-r\left(t_{2}\right)\left[\rho_{1} \rho_{2}-\tau^{2}\right]}{\left[1-r\left(t_{1}\right) \rho_{1}\right]\left[1-r\left(t_{2}\right) \rho_{2}\right]-r\left(t_{1}\right) r\left(t_{2}\right) \tau^{2}} .
\end{aligned}
$$


The print's global transmittance $T$ is derived from Eq. (3) for light penetrating through the recto-colored interface and emerging through the verso-colored interface:

$$
\begin{aligned}
T= & T_{\mathrm{in}}\left(\theta_{0}\right) T_{\mathrm{ex}}\left(\theta_{0}^{\prime \prime}\right) T_{m} \\
= & T_{01}\left(\theta_{0}\right) \frac{T_{01}\left(\theta_{0}^{\prime \prime}\right)}{\pi n^{2}} t_{1}^{\mu\left(\theta_{0}\right)} t_{2}^{\mu\left(\theta_{0}^{\prime \prime}\right)} \\
& \times \frac{\tau}{\left[1-r\left(t_{1}\right) \rho_{1}\right]\left[1-r\left(t_{2}\right) \rho_{2}\right]-r\left(t_{1}\right) r\left(t_{2}\right) \tau^{2}} .
\end{aligned}
$$

Other specific expressions of $R$ and $T$ are obtained for a diffuse illumination or when the emerging light is captured with an integrating sphere. The terms $T_{\text {in }}$ and $T_{\text {ex }}$ are given, respectively, by Eqs. (28) and (35). If a halftone color is printed on the recto and/or the verso, the inked layer is composed of several colorants of respective transmittances $t_{k}$ and surface coverages $a_{k}$. The expression of $T_{\text {in }}$ is specified by Eq. (26) for a collimated illumination, or approximation (29) for a diffuse illumination. The expression of $T_{\text {ex }}$ is specified by Eq. (31) for a radiance detector, or approximation (36) for an integrating sphere. The internal reflectances $r_{1}$ and $r_{2}$ of the colored interfaces are expressed according to Eq. (23).

Note that $45^{\circ} 0^{\circ}$ photospectrometers measure reflectance factors, which are defined with respect to a reference white Lambertian diffuser of reflectance $1 / \pi$, i.e., it is the reflectance of Eq. (44) multiplied by a factor $\pi$. The same applies to transmission factors measured with photospectrometers at an angle of $0^{\circ}$.

\section{INTERNAL REFLECTANCE AND TRANSMITTANCE OF THE PRINT: KUBELKA-MUNK THEORY EXTENDED TO RECTO-VERSO HALFTONE PRINTS}

The internal reflectance $R_{m}$ and transmittance $T_{m}$ of a recto-verso halftone print can also be obtained by describing the light scattering within the substrate layer thanks to the Kubelka-Munk theory. Light scattering is described by a system of differential equations whose solutions represent the irradiance propagating upwards and the irradiance propagating downwards as functions of the depth within the substrate layer. The reflectance of the substrate is the ratio of the upward irradiance at the depth 0 (recto substrate layer boundary) to the incident irradiance. Its transmittance is the ratio of the downward irradiance at the depth $h$ (the verso substrate layer boundary) to the incident irradiance.

The original Kubelka-Munk model was formulated for diffusing layers bounded at the verso by a reflecting background. ${ }^{7}$ The internal reflection of light at the recto interface, i.e., the interface between the substrate and the air, was ignored. Saunderson introduced a correction that accounts for the light reflection at the recto interface. ${ }^{9}$ The classical methods used to solve the Kubelka-Munk differential equation system require the introduction of boundary conditions. In the present contribution, we rely on the Laplace transform to calculate very simply the general solutions of the Kubelka-Munk differential equation system independently of the boundary conditions (see Appendix C).
We then specify the boundary conditions according to the optical elements bounding the scattering layer. The boundary conditions yield one relation between the upward and the downward irradiances at depth 0 and a second relation at depth $h$. We insert these relations into the general solutions of the differential equation system, and derive the irradiances reflected and transmitted by the scattering layer and its boundaries. This yields the internal reflectance and the internal transmittance of the bounded scattering layer. In Appendix D, we consider the case where the scattering layer is bounded with reflectors at its two sides. The original Kubelka-Munk expressions for the reflectance of a layer superposed on top of a background and the Saunderson correction are derived in Appendix $\mathrm{E}$ as special cases of the expressions of our new formulation of the Kubelka-Munk model.

In the present section, we apply the Kubelka-Munk model extended with recto and verso reflectors to the specific case where the reflectors are interfaces colored with halftone inks. By establishing the relation between the parameters of our multiple reflection-transmission model and those of the Kubelka-Munk model, we show that both approaches are equivalent. The respective advantages of the two approaches are presented at the end of this section.

\section{A. Kubelka-Munk Differential Equations}

The substrate is characterized by a wavelengthdependent absorption coefficient $K(\lambda)$ and a wavelengthdependent scattering coefficient $S(\lambda)$. The printing support is a substrate layer of thickness $h$. In this layer, a diffuse irradiance $i_{r}$ propagates upwards and a diffuse irradiance $i_{t}$ propagates downwards. Both $i_{r}$ and $i_{t}$ are functions of the depth $x$ within the substrate layer. The depth 0 corresponds to the substrate layer's boundary that receives the incident irradiance $I_{0}$ (Fig. 3). The depth $h$ corresponds to the other substrate layer's boundary.

Let us consider a certain depth $x$ within the substrate layer. Between the depths $x$ and $x+\mathrm{d} x$, the downward irradiance $i_{t}$ loses a fraction $K \mathrm{~d} x$ of its irradiance through absorption and a fraction $S \mathrm{~d} x$ due to backscattering. It receives a fraction $S \mathrm{~d} x$ of the backscattered upward irradiance $i_{r}$. We have

$$
i_{t}(x+\mathrm{d} x)=i_{t}(x)-(K+S) i_{t}(x) \mathrm{d} x+S i_{r}(x) \mathrm{d} x .
$$

Between the depths $x$ and $x-\mathrm{d} x$, the upward irradiance $i_{r}$ loses a fraction $K \mathrm{~d} x$ of its irradiance and a fraction $S \mathrm{~d} x$

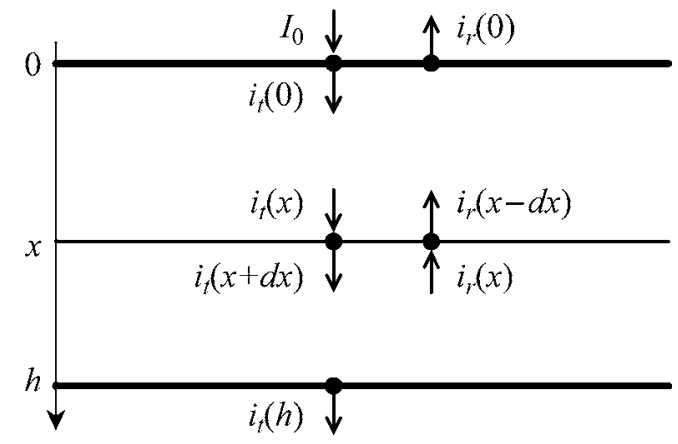

Fig. 3. Upward and downward irradiances crossing a sublayer of thickness $\mathrm{d} x$ at a depth $x$ in the substrate layer. 
due to backscattering. It receives a fraction $S \mathrm{~d} x$ of the backscattered downward irradiance $i_{t}$. We have

$$
i_{r}(x-\mathrm{d} x)=i_{r}(x)-(K+S) i_{r}(x) \mathrm{d} x+S i_{t}(x) \mathrm{d} x .
$$

According to the definition of the derivative

$$
\frac{\mathrm{d}}{\mathrm{d} x} i=\frac{i(x)-i(x-\mathrm{d} x)}{\mathrm{d} x}=\frac{i(x+\mathrm{d} x)-i(x)}{\mathrm{d} x},
$$

we rearrange Eqs. (46) and (47) and obtain the KubelkaMunk differential equation system

$$
\begin{aligned}
& \frac{\mathrm{d}}{\mathrm{d} x} i_{r}(x)=(K+S) i_{r}(x)-S i_{t}(x), \\
& \frac{\mathrm{d}}{\mathrm{d} x} i_{t}(x)=S i_{r}(x)-(K+S) i_{t}(x) .
\end{aligned}
$$

The general solutions $i_{r}(x)$ and $i_{t}(x)$ of the differential equation system (48) can be determined by applying the Laplace transform without having to introduce boundary conditions. The Laplace transform enables us to convert the differential equation system into a linear equation system (Appendix C).

\section{B. Internal Reflectance and Transmittance of the Print} The print's internal reflectance $R_{m}$ and transmittance $T_{m}$ are the ratios of the exiting irradiances $i_{r}(0)$ and $i_{t}(h)$, respectively, to the incident irradiance $I_{0}$. To obtain $i_{r}(0)$ and $i_{t}(h)$, we introduce boundary conditions with respect to the reflectors that bound the substrate layer at $x=0$ and $x=h$. In Appendix D, we consider that the substrate layer is bounded at $x=0$ by a reflector of reflectance $r_{0}$ and at $x=h$ by a reflector of reflectance $r_{h}$. We obtain an expression for $i_{r}(0)$ and $i_{t}(h)$ and derive both the internal reflectance $R_{b}$ [Eq. (D7)] and the internal transmittance $T_{b}$ [Eq. (D8)] of the bounded substrate layer.

In the present case, the reflectors are the recto and the verso interfaces incorporating each one their respective inked layers. At $x=0$, the recto colored interface has a reflectance $r_{1}$ [Eq. (23)]. At $x=h$, the verso colored interface has a reflectance $r_{2}$. The print's internal reflectance $R_{m}$ and transmittance $T_{m}$ are identical to the expressions of $R_{b}$ and $T_{b}$ [Appendix D, Eqs. (D7) and (D8)], with $r_{0}=r_{1}$ and $r_{h}=r_{2}$ :

$$
R_{m}=\frac{\left(1-a r_{2}\right) \sinh +b r_{2} \cosh (b S h)}{\left(a-r_{1}-r_{2}+a r_{1} r_{2}\right) \sinh (b S h)+b\left(1-r_{1} r_{2}\right) \cosh (b S h)},
$$

$$
T_{m}=\frac{b}{\left(a-r_{1}-r_{2}+a r_{1} r_{2}\right) \sinh (b S h)+b\left(1-r_{1} r_{2}\right) \cosh (b S h)},
$$

with

$$
a=\frac{K+S}{S}, \quad b=-\sqrt{a^{2}-1} .
$$

\section{Intrinsic Reflectance and Transmittance of the Substrate Layer}

Let us express the intrinsic reflectance $\rho$ and transmittance $\tau$ of the substrate layer. We consider the substrate layer as a print that is not bounded by any reflector. The reflectance and the transmittance of the print without reflectors are given by Eqs. (D7) and (D8) with $r_{0}=r_{h}=0$ :

$$
\rho=\frac{\sinh (b S h)}{b \cosh (b S h)+a \sinh (b S h)},
$$

$$
\tau=\frac{b}{b \cosh (b S h)+a \sinh (b S h)} .
$$

These expressions are identical to the expressions derived by Kubelka. ${ }^{7}$

\section{Comparison between our Model and the Kubelka-Munk Model Extended to Recto-Verso Halftone Prints}

The print's internal reflectance $R_{m}$ and transmittance $T_{m}$ have been expressed, for a symmetric printing support, with the multiple reflection-transmission model in Eqs. (42) and (43). The substrate was characterized by its intrinsic reflectance $\rho$ and its intrinsic transmittance $\tau$. In Eqs. (51) and (52), we express $\rho$ and $\tau$ according to the Kubelka-Munk model as functions of the absorption coefficient $K$, the scattering coefficient $S$, and the substrate layer thickness $h$. Let us replace in Eqs. (42) and (43) $\rho$ and $\tau$ by their expressions given in Eqs. (51) and (52). We obtain the same expressions for the internal reflectance $R_{m}$ and the internal transmittance $T_{m}$ as the ones obtained in Eqs. (49) and (50) with the extended KubelkaMunk model. This shows the equivalence between our multiple reflection-transmission model and the extension of Kubelka-Munk's model to a scattering layer bounded by two colored interfaces.

Both models assume that the substrate is a strongly scattering medium. In the case of halftone prints, they also assume that the lateral propagation of light within the substrate is large with respect to the screen element period, i.e., the attenuation of light traversing an inked layer is proportional to the sum of the colorant transmittances weighted by their surface coverages.

However, in contrast to the Kubelka-Munk model, the multiple reflection-transmission model is capable of predicting reflectances and transmittances for nonsymmetric printing supports, or for complex multilayer supports. The model may be applied to a print support composed of several superposed layers, comprising at the center nonscattering or weekly scattering layers. The only condition is that the exterior layers are strongly scattering, ensuring that light is completely diffused as soon as it penetrates the support and that the print's interfaces with the air are illuminated from their print side by Lambertian irradiance. The recto intrinsic reflectance, the verso intrinsic reflectance, and the intrinsic transmittance of the substrate can be deduced from spectral measurements. 


\section{RELATION TO THE WILLIAMS-CLAPPER AND THE CLAPPER-YULE MODELS}

The Williams-Clapper model enables us to predict the reflectance of a nonscattering solid colorant layer superposed on a diffusing background ${ }^{6}$ of the same refractive index. The background is composed of a substrate of reflectance $\rho_{B}$. The original Williams-Clapper model relying on collimated $45^{\circ}$ illumination and $0^{\circ}$ radiance capture was extended to any illuminating and capturing geometries. $^{26,27}$

Let us consider a colorant layer of normal transmittance $t$ (attenuation of a light ray crossing the colorant layer along its normal direction), a collimated illumination at an angle $\theta_{0}$, and a radiance detector at an angle $\theta_{0}^{\prime}$. The reflectance is expressed according to the extended Williams-Clapper model [Ref. 27, Eq. (44)] as

$$
R_{\mathrm{WC}}=T_{01}\left(\theta_{0}\right) \frac{T_{01}\left(\theta_{0}^{\prime}\right)}{\pi n^{2}} \frac{\rho_{B} t^{1 / \cos \theta_{0}} t^{1 / \cos \theta_{0}^{\prime}}}{1-\rho_{B} r(t)}
$$

where $r(t)$ is the diffuse reflectance of the colored interface [Eq. (22)]. Note that in the Williams-Clapper model, the reflectance factor is defined with respect to a reference white perfect diffuser of reflectance $1 / \pi$, i.e., it is the reflectance multiplied by a factor $\pi$.

Let us establish the relation between the substrate reflectance $\rho_{B}$ of the Williams-Clapper model and the substrate's intrinsic parameters of our multiple reflectiontransmission model (recto intrinsic reflectance $\rho_{1}$, verso intrinsic reflectance $\rho_{2}$, and intrinsic transmittance $\tau$ ). Obviously, if the substrate layer is perfectly opaque, no light can reach the verso. If the substrate has no verso interface, the light crossing the substrate exits at the verso and does not contribute to the substrate reflectance $\rho_{B}$, as if its intrinsic transmittance would be $\tau=0$. In that case, the substrate reflectance $\rho_{B}$ is equal to its recto intrinsic reflectance $\rho_{1}$, i.e., $\rho_{B}=\rho_{1}$. On the contrary, if the substrate has a verso interface, the light transmitted across the substrate may be reflected at this interface, cross the substrate again, and contribute to the substrate reflectance $\rho_{B}$. To establish the relation among $\rho_{B}, \rho_{1}, \rho_{2}$, and $\tau$, we consider a downward incident irradiance $W_{i}$ initially located just beneath the recto interface. We describe the interaction of this irradiance with the grounded substrate, i.e., with the substrate and its verso, and calculate the total irradiance $W_{r}$ that eventually reaches the recto interface. The verso part of the grounded substrate has a reflectance $r_{2}$. It is either the verso interface alone, of reflectance $r_{2}=r_{10}$, or a colored interface of reflectance $r_{2}$ $=r\left(t_{2}\right), t_{2}$ being the normal transmittance of the verso inked layer (Section 4).

Figure 4(b) describes the interaction of the incident irradiance $W_{i}$ with the substrate and the verso. A fraction $\rho_{1}$ of $W_{i}$ is directly reflected by the substrate without reaching the verso. A fraction $\tau$ of $W_{i}$ crosses the interface and reaches the verso where a fraction $r_{2}$ is reflected. The reflected irradiance $\pi r_{2} W_{i}$ either crosses the substrate back to the recto within a proportion $\tau$ or undergoes several reflections between the substrate (intrinsic reflectance $\rho_{2}$ ) and the verso-colored interface (reflectance $r_{2}$ ) recto

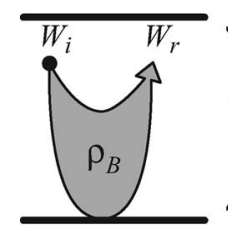

(a)

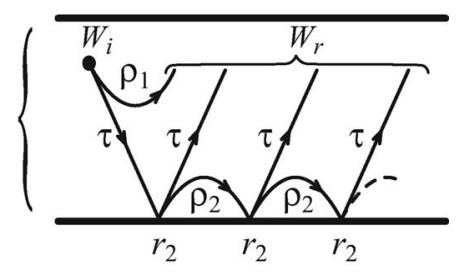

(b)
Fig. 4. (a) Grounded substrate reflectance $\rho_{B}$, as defined in the Williams-Clapper model, is the ratio between the irradiance $W_{r}$ reflected by the substrate bounded on its verso and the incident irradiance $W_{i}$. (b) The reflected irradiance $W_{r}$ can be expressed as a function of the substrate's intrinsic reflectances ( $\rho_{1}$ at the recto side, $\rho_{2}$ at the verso side) and its intrinsic transmittance $\tau$ by taking into account the multiple internal reflections at the verso interface (reflectance $r_{2}$ ).

before crossing the substrate toward the recto. The total irradiance $W_{r}$ reaching the recto is

$$
W_{r}=\rho_{1} W_{i}+\tau^{2} r_{2} W_{i}\left(1+r_{2} \rho_{2}+r_{2}^{2} \rho_{2}^{2}+\ldots\right) .
$$

The infinite sum forms a geometric series converging toward $1 /\left(1-r_{2} \rho_{2}\right)$. The reflected irradiance becomes

$$
W_{r}=\left(\rho_{1}+\frac{r_{2} \tau^{2}}{1-r_{2} \rho_{2}}\right) W_{i},
$$

and the grounded substrate reflectance $\rho_{B}$ is

$$
\rho_{B}=\frac{W_{r}}{W_{i}}=\rho_{1}+\frac{r_{2} \tau^{2}}{1-r_{2} \rho_{2}} .
$$

The same expression for $\rho_{B}$ would be given by Kubelka's multilayer model [Ref. 8, Eq. (10)] if $r_{2}$ was the reflectance of a second scattering layer of the same refractive index.

In the Williams-Clapper reflectance expression [Eq. (53)], we replace the reflectance of the grounded substrate layer $\rho_{B}$ by its expanded expression [Eq. (54)] and obtain

$$
\begin{aligned}
R_{\mathrm{WC}}= & T_{01}\left(\theta_{0}\right) \frac{T_{01}\left(\theta_{0}^{\prime}\right)}{\pi n^{2}} t^{1 / \cos \theta_{0}} t^{t / \cos \theta_{0}^{\prime}} \\
& \times \frac{\rho_{1}-r_{2}\left(\rho_{1} \rho_{2}-\tau^{2}\right)}{\left[1-r(t) \rho_{1}\right]\left(1-\rho_{2} r_{2}\right)-r(t) r_{2} \tau^{2}} .
\end{aligned}
$$

This is the same expression as the one derived according to our recto-verso model [Eq. (44)]. Therefore, the Williams-Clapper model is a particular embodiment of our recto-verso model where the Williams-Clapper substrate reflectance $\rho_{B}$ accounts for the multiple internal reflections between the substrate and the verso paper side.

Another classical model, the Clapper-Yule model, predicts the reflectance of halftone prints when the lateral propagation of light within the substrate is large with respect to the screen element period. ${ }^{5,27}$ Both the ClapperYule model and our recto-verso model rely on the same assumptions regarding the lateral propagation of light within the substrate and the uniform thickness of the ink. As in the Williams-Clapper model, the printing support is a background of reflectance $\rho_{B}$. The reflectance $R_{\mathrm{CY}}$ of a 
print illuminated under an angle $\theta_{0}$ and observed by a radiance detector under an angle $\theta_{0}^{\prime}$ is, according to the Clapper-Yule model [Ref. 27, Eq. (59)],

$$
R_{\mathrm{CY}}=T_{01}\left(\theta_{0}\right) \frac{T_{01}\left(\theta_{0}^{\prime}\right)}{\pi n^{2}} \frac{\rho_{B}\left(\sum a_{k} t_{k}\right)^{2}}{1-\rho_{B} r_{10} \sum a_{k} t_{k}^{2}} .
$$

Equation (56) can be decomposed into a light penetration attenuation $T_{\text {in }}$, an internal reflectance $R_{m}$, and a light emergence attenuation $T_{\mathrm{ex}}$, as in Eq. (2), with

$$
\begin{aligned}
& T_{\text {in }}=T_{01}\left(\theta_{0}\right) \sum a_{k} t_{k}, \\
& T_{\text {ex }}=\frac{T_{01}\left(\theta_{0}^{\prime}\right)}{\pi n^{2}} \sum a_{k} t_{k}, \\
& R_{m}=\frac{\rho_{B}}{1-\rho_{B} r_{10} \sum a_{k} t_{k}^{2}} .
\end{aligned}
$$

In contrast to the Williams-Clapper model and to our recto-verso model, the Clapper-Yule model ignores the fact that the incident and emerging light may follow an oblique path within the inked layers. ${ }^{27}$ Therefore, the exponents $\mu\left(\theta_{0}\right)$ and $\mu\left(\theta_{0}^{\prime}\right)$ applied to the ink transmittance $t_{k}$ are ignored in Eqs. (57) and (58).

In Eq. (59) let us replace $r_{10} \Sigma a_{k} t_{k}^{2}$ by $r_{1}$ and the background reflectance $\rho_{B}$ by Eq. (54). The print's internal reflectance becomes

$$
\begin{aligned}
R_{m} & =\frac{\rho_{1}\left(1-\rho_{2} r_{2}\right)+r_{2} \tau^{2}}{\left(1-\rho_{2} r_{2}\right)-\left[\left(1-\rho_{2} r_{2}\right) \rho_{1}+r_{2} \tau^{2}\right]} \\
& =\frac{\rho_{1}-r_{2}\left(\rho_{1} \rho_{2}-\tau^{2}\right)}{\left(1-r_{1} \rho_{1}\right)\left(1-r_{2} \rho_{2}\right)-r_{1} r_{2} \tau^{2}} .
\end{aligned}
$$

The print's internal reflectance $R_{m}$ obtained according to our model [Eq. (40)] and according to the Clapper-Yule model [Eq. (60)] have a similar expression. A small difference exists between the two models in the detailed expression of $r_{1}$. The expression $r_{10} \sum a_{k} t_{k}^{2}$ used in the Clapper-Yule model is an approximation of Eq. (23) used in our model. The Clapper-Yule model, for a given colorant of transmittance $t_{k}$, makes implicitly the following approximation $^{27}$ :

$$
r\left(t_{k}\right) \approx r_{10} t_{k}^{2}
$$

i.e.,

$$
\int_{\theta=0}^{\pi / 2} t_{k}^{t / \cos \theta} R_{10}(\theta) \sin 2 \theta \mathrm{d} \theta \approx t_{k}^{2} \int_{\theta=0}^{\pi / 2} R_{10}(\theta) \sin 2 \theta \mathrm{d} \theta .
$$

In contrast to our recto-verso model, the WilliamsClapper and the Clapper-Yule models include the reflecting properties of the verso (reflections at the verso-colored interface and absorption within a verso inked layer) into the grounded substrate reflectance $\rho_{B}$. Each time a new color is printed at the verso, parameter $\rho_{B}$ has to be measured again. These classical models are therefore reflectance models for recto-only prints. In contrast to the Williams-Clapper and the Clapper-Yule models, our model is a reflectance and transmittance recto-verso print prediction model. We can predict the print's reflectance spectrum for any combination of recto-verso colors by measuring and deducing the recto and verso intrinsic reflectances $\rho_{1}$ and $\rho_{2}$ as well as the intrinsic transmittance $\tau$ only once.

\section{CALIBRATING THE RECTO-VERSO REFLECTANCE AND TRANSMITTANCE MODEL}

To predict the reflectance and transmittance spectrum of recto-verso halftone prints, we first calibrate the model by calculating all the parameters characterizing the selected printing support and the selected inks: the recto and verso intrinsic reflectances $\rho_{1}(\lambda)$ and $\rho_{2}(\lambda)$ of the substrate layer, its intrinsic transmittance $\tau(\lambda)$, the transmittance $t_{i}(\lambda)$ of the colorants, and the surface coverage $a$ of each colorant on the recto and on the verso. The colorants effective surface coverages are estimated as functions of the nominal surface coverages. ${ }^{14}$ The parameters are calculated thanks to reflectance and transmittance measurements performed on calibration samples. We assume that the incident light is collimated and oriented, with an incidence angle $\theta_{0}$. The emerging light is also captured in a single direction with an angle $\theta_{0}^{\prime}$ for the reflectance and $\theta_{0}^{\prime \prime}$ for the transmittance. In the present contribution, we consider halftone patches printed with a single color ink, e.g., cyan, magenta, or yellow. However, by applying the Demichel equations ${ }^{28}$ and by taking into account ink spreading that occurs when an ink halftone is printed on top of another ink, ${ }^{14}$ we may apply the model to polychromatic halftones.

The first calibration sample corresponds to an unprinted area of the printing support. We measure its reflectance $R_{1}(\lambda)$, whose expression is given by Eq. (44), with $t=1$ [the reflectance of the interfaces $r(t)$ becomes $\left.r_{10}\right]$. We also measure the transmittance $T(\lambda)$ of that sample, given by Eq. (45) with $t=1$. Then we measure the reflectance $R_{2}(\lambda)$ of the same sample upside down whose expression is the same as $R_{1}(\lambda)$, but with inverted intrinsic reflectances $\rho_{1}$ and $\rho_{2}$. These measurements allow us to deduce the spectral intrinsic reflectances $\rho_{1}$ and $\rho_{2}$ and the spectral intrinsic transmittance $\tau$ by solving numerically the system of Eqs. (62) for each wavelength:

$$
\begin{gathered}
R_{1}=T_{01}\left(\theta_{0}\right) \frac{T_{01}\left(\theta_{0}^{\prime}\right)}{\pi n^{2}} \frac{\rho_{1}-r_{10}\left(\rho_{1} \rho_{2}-\tau^{2}\right)}{\left(1-r_{10} \rho_{1}\right)\left(1-r_{10} \rho_{2}\right)-\left(r_{10} \tau\right)^{2}}, \\
R_{2}=T_{01}\left(\theta_{0}\right) \frac{T_{01}\left(\theta_{0}^{\prime}\right)}{\pi n^{2}} \frac{\rho_{2}-r_{10}\left(\rho_{1} \rho_{2}-\tau^{2}\right)}{\left(1-r_{10} \rho_{1}\right)\left(1-r_{10} \rho_{2}\right)-\left(r_{10} \tau\right)^{2}}, \\
T=T_{01}\left(\theta_{0}\right) \frac{T_{01}\left(\theta_{0}^{\prime}\right)}{\pi n^{2}} \frac{\tau}{\left(1-r_{10} \rho_{1}\right)\left(1-r_{10} \rho_{2}\right)-\left(\rho_{10} \tau\right)^{2}} .
\end{gathered}
$$

The terms $T_{01}\left(\theta_{0}\right), T_{01}\left(\theta_{0}^{\prime}\right)$, and $r_{10}$ are known since they depend only on the print's relative refractive index $n$, typically equal to 1.5 for a print in contact with air (see Appendix A). 
To deduce solid ink transmittances, we now consider a calibration sample covered on the recto with a single solid ink layer. We measure its reflectance and its transmittance. Their expressions are given, respectively, by Eqs. (44) and (45), with $t$ being the transmittance of the solid ink layer, with $r_{2}=r_{10}$ being the diffuse reflectance of the verso interface and with $r(t)$ being the diffuse reflectance of the recto-colored interface, expressed by Eq. (22) and tabulated in Table 2 (see Appendix A). We obtain

$R$

$$
=T_{01}\left(\theta_{0}\right) \frac{T_{01}\left(\theta_{0}^{\prime}\right)}{\pi n^{2}} t^{\mu\left(\theta_{0}\right)+\mu\left(\theta_{0}^{\prime}\right)} \frac{\rho_{1}-r_{10}\left(\rho_{1} \rho_{2}-\tau^{2}\right)}{\left(1-r(t) \rho_{1}\right)\left(1-r_{10} \rho_{2}\right)-r_{10} r(t) \tau^{2}},
$$

$$
T=T_{01}\left(\theta_{0}\right) \frac{T_{01}\left(\theta_{0}^{\prime \prime}\right)}{\pi n^{2}} t^{\mu\left(\theta_{0}\right)} \frac{\tau}{\left(1-r(t) \rho_{1}\right)\left(1-r_{10} \rho_{2}\right)-r_{10} r(t) \tau^{2}}
$$

We assume that the exponent function $\mu(\theta)$ has been evaluated numerically (see Appendix B). Since $\rho_{1}, \rho_{2}$, and $\tau$ have also been deduced from Eqs. (62), Eqs. (63) and (64) depend only on the ink transmittance $t$, which can be computed numerically for each wavelength. We obtain one spectral ink transmittance from the measure of reflectance and another one from the measure of transmittance. In theory, these two ink transmittance spectra should be equal.

By repeating this procedure for each ink, and for each combination of two and three superposed solid inks, we obtain the spectral transmittances $t_{c}, t_{m}, t_{y}, t_{r}, t_{g}, t_{b}, t_{k}$ of the respective colorants cyan, magenta, yellow, red, green, blue, and black. The transmittance of the colorants printed on the verso are evaluated separately by following the same procedure as above.

In exceptional cases, inks and substrate surfaces are identical on the recto and verso side of the print, yielding identical colorant transmittances. In practice, however, we observe a small difference between the ink transmittance spectra derived from the measure of the print reflectance and the measure of the print transmittance. Since our model assumes that the inks are nonscattering, the difference may originate from the backscattering of light within the inked layers.

We now consider single ink halftone patches printed at different nominal surface coverages $a$, e.g., 25\%, 50\%, $75 \%$. The inked layer is composed of two colorants: The first colorant corresponds to the ink (transmittance $t$ ) and the second one corresponds to the paper white, i.e., no ink (transmittance 1 and coverage $1-a$ ). Because of the ink spreading phenomenon (mechanical dot gain), the physical surface coverage $a$ of an ink halftone differs from its nominal coverage. We measure the reflectance and the transmittance of each patch. Their expressions are given by Eqs. (2) and (3), with the following expressions for the attenuation factor $T_{\text {in }}$ of the penetrating light, for the attenuation factor $T_{\mathrm{ex}}$ of the emerging light, and for the print's internal reflectance $R_{m}$ and internal transmittance $T_{m}$ :
- $T_{\text {in }}$ is expressed according to Eq. (26) with the two colorants paper white and ink:

$$
T_{\text {in }}=T_{01}\left(\theta_{0}\right)\left(1-a+a t^{\mu\left(\theta_{0}\right)}\right) .
$$

- For the print reflectance, since light emerges across the colored recto interface, $T_{\text {ex }}$ derives from Eq. (31), with the two colorants paper white and ink:

$$
T_{\mathrm{ex}}=\frac{T_{01}\left(\theta_{0}^{\prime}\right)}{\pi n^{2}}\left(1-a+a t^{\mu\left(\theta_{0}^{\prime}\right)}\right) .
$$

- For the print transmittance, since light emerges across the unprinted verso interface, $T_{\mathrm{ex}}$ derives from Eq. (30) with $t=1$ :

$$
T_{\mathrm{ex}}^{\prime}=\frac{T_{01}\left(\theta_{0}^{\prime}\right)}{\pi n^{2}}
$$

- $R_{m}$ and $T_{m}$ are expressed by Eqs. (40) and (41), where the diffuse reflectance of the verso interface is $r_{2}=r_{10}$, and where the diffuse reflectance of the recto-colored interface $r_{1}$ derives from Eq. (23) with the two colorants paper white and ink:

$$
r_{1}=(1-a) r_{10}+a r(t)
$$

where $r_{10}$ is the diffuse reflectance of the paper white colored interface surface part and $r(t)$ is the diffuse reflectance of the ink colored interface surface part [Eq. (22)].

From the reflectance expression or from the transmittance expression we fit the unknown physical coverage $a$ by minimizing the sum of square differences between the measured and the predicted spectra. For each ink, we obtain a table of correspondence between the nominal and the effective surface coverages. Thanks to linear interpolation, tabulated values are converted to a continuous curve. Nominal to effective surface coverage curves are also calculated for the inks printed on the verso.

\section{EXPERIMENTAL VERIFICATION}

We have tested the reflectance and transmittance prediction model on recto-verso halftone patches printed at 180 lines per inch with an offset proofing press. The patches were printed on white polyvinyl chloride sheets (titanium oxide pigments). This printing support has perfectly flat interfaces, is not fluorescent, does not allow the inks to penetrate the substrate, and has a refractive index of 1.54. It thus satisfies the properties required to apply the recto-verso reflectance and transmittance prediction model.

Since the print is assumed to be only in contact with the air, we raised the printing support a few centimeters above the table.

The model was calibrated from transmittance measurements according to the method presented in Section 8 . Since the recto and the verso have been printed during different offset print passes, respective ink thicknesses and surface coverages are slightly different between the recto and the verso. We therefore calibrate separately the recto and the verso inked layers. The parameters of the 
Table 1. Difference in $\Delta$ E94 between Predicted and Measured Transmittance Spectra ${ }^{a}$

\begin{tabular}{|c|c|c|c|c|c|c|c|c|c|}
\hline \multicolumn{10}{|c|}{ Inked Layer on Verso $^{b}$} \\
\hline Recto $^{b}$ & $\mathrm{C}$ & $\mathrm{M}$ & $\mathrm{Y}$ & $\mathrm{R}$ & $\mathrm{G}$ & B & $\mathrm{K}$ & C50 & Average $^{c}$ \\
\hline $\mathrm{C}$ & 0.5 & 1.2 & 2.8 & 0.6 & 1.3 & 0.6 & 1.6 & 1.5 & 1.3 \\
\hline M & 2.2 & 1.2 & 0.9 & 0.9 & 1.3 & 0.9 & 1.6 & 0.9 & 1.2 \\
\hline $\mathrm{Y}$ & 1.0 & 0.7 & 0.5 & 0.8 & 0.9 & 0.3 & 1.6 & 1.3 & 0.9 \\
\hline $\mathrm{R}$ & 2.3 & 0.8 & 0.4 & 0.7 & 1.5 & 1.1 & 1.4 & 0.4 & 1.1 \\
\hline $\mathrm{G}$ & 0.5 & 0.5 & 2.6 & 0.7 & 0.8 & 0.4 & 3.3 & 2.3 & 1.4 \\
\hline B & 2.1 & 1.0 & 0.2 & 1.8 & 1.6 & 1.2 & 1.1 & 1.4 & 1.3 \\
\hline $\mathrm{K}$ & 2.0 & 1.6 & 0.4 & 1.5 & 0.7 & 1.5 & 1.4 & 1.7 & 1.4 \\
\hline $\mathrm{C} 25$ & 0.6 & 2.3 & 0.7 & 2.0 & 2.3 & 0.7 & 1.7 & 0.7 & 1.4 \\
\hline C50 & 0.5 & 2.0 & 1.6 & 1.9 & 2.0 & 1.1 & 2.5 & 0.8 & 1.6 \\
\hline $\mathrm{C} 75$ & 0.2 & 2.0 & 1.7 & 1.6 & 1.5 & 0.7 & 1.5 & 0.5 & 1.2 \\
\hline Average $^{c}$ & 1.2 & 1.3 & 1.2 & 1.3 & 1.4 & 0.9 & 1.8 & 1.2 & 1.3 \\
\hline
\end{tabular}

${ }^{a}$ For offset inks printed on a polyvinyl chloride substrate. Differences between transmittance spectra are expressed as CIELAB $\Delta$ E94 values.

${ }^{b} \mathrm{C}$ stands for cyan solid inked layer; $\mathrm{M}$ for magenta; $\mathrm{Y}$ for yellow; R for superposed magenta and yellow solid inks yielding the red colorant; $\mathrm{G}$ for solid yellow and cyan yielding the green colorant; B for solid cyan and magenta yielding the blue colorant; K for the superposed cyan, magenta, and yellow solid inks, yielding the black colorant. C25, C50, and C75 stand for cyan halftones of respective nominal surface coverage $0.25,0.5$, and 0.75 .

${ }^{c}$ Average of the $\Delta$ E94 values of the corresponding column or row in the table.

recto inked layer are calculated from patches printed on the recto only (unprinted verso), and those of the verso inked layer are calculated from patches printed on the verso only (unprinted recto). We predict the transmittance spectra of recto-verso patches. The difference between the predicted and the measured spectra (Table 1) is expressed according to the CIELAB $\Delta \mathrm{E} 94$ color difference formulas. ${ }^{30}$

The mean difference between measured transmission spectra and transmission spectra predicted according to the recto-verso model is $\Delta \mathrm{E} 94=1.3$. As can be seen from Table 1, more than $95 \%$ of the predicted transmittance spectra have a $\Delta \mathrm{E} 94$ prediction error of less than 2.5, which corresponds to the normal offset printing accuracy. This experiment shows that the prediction accuracy that is achieved by the proposed model is similar to the prediction accuracy of reflection spectra that is achieved by the classical Clapper-Yule model ${ }^{14}$ for recto-only offset prints.

\section{CONCLUSIONS}

We present a new model enabling us to predict both the reflectance and the transmittance of recto-verso halftone prints. Both sides of the print are allowed to be printed with different solid or halftone inks. The model assumes that light propagation within the print is important with respect to the halftone screen period. The model comprises three independent components. The two components describing the attenuation of incident and exiting light take into account the Fresnel transmission across the interface and the absorption by the inked layer, according to, respectively, the geometry of illumination and the geometry of light detection. We consider the cases of collimated and of perfectly diffuse illumination. With respect to light detection, we consider the cases of the integrating sphere and of the radiance detector. The third model component, which is independent of the illumina- tion and detection conditions, accounts for the multiple internal reflections between the interfaces and the diffusing substrate, as well as for the multiple transmissions from one interface to the other across the substrate. This third model component yields one expression for the recto-verso halftone print internal reflectance and one expression for its internal transmittance.

Both the internal reflectance and the internal transmittance can also be obtained by extending the KubelkaMunk model to recto-verso inked interfaces. We establish a relationship between the internal reflectance and transmittance of our model and Kubelka-Munk's scattering and absorption parameters. By deducing the internal reflectance or transmittance of our model from spectral measurements, one may easily compute the corresponding Kubelka-Munk scattering and absorption parameters. The recto-verso reflectance and transmittance model is, however, more general than the Kubelka-Munk model, since it does not assume a homogeneous scattering substrate. The only condition is that the exterior layers are strongly scattering, ensuring that light is completely diffuse when penetrating or emerging from the substrate. Both the recto intrinsic reflectance and the verso intrinsic reflectance, and therefore the corresponding internal reflectance, can be easily deduced from spectral reflectance measurements carried out on the two sides of the recto-verso print.

Since the proposed reflectance and transmittance model supports the classical Williams-Clapper and the Clapper-Yule models as special cases and since it encompasses the extended Kubelka-Munk model, it provides a unified model for scattering substrates with inked interfaces. Such a unified model offers new perspectives both for spectral reflection and transmission predictions and for characterizing the properties of both color halftone prints and scattering materials painted with nonscattering inks. 


\section{APPENDIX A: NUMERICAL EVALUATIONS OF FRESNEL REFLECTANCES AND TRANSMITTANCES}

The fractions of incident light reflected and transmitted by a print-air interface depend on the relative index of refraction of the interface, the side on which the light is incident, and on the angular distribution of the incident light. Most printing supports have a refractive index very close to $n_{1}=1.5$. The refractive index of the air $n_{0}$ is equal to 1 . We give here the numerical values of the reflectance and transmittance of a print-air interface whose relative index of refraction is $n_{1} / n_{0}=1.5$.

Let us first consider that the incident light is collimated. The reflectance and the transmittance of the interface are given by the Fresnel coefficients. According to the notations introduced in Section 2, the reflectance and the transmittance of the interface illuminated from the air under an incidence $\theta_{0}$ are, respectively, $R_{01}\left(\theta_{0}\right)$ and $T_{01}\left(\theta_{0}\right)=1-R_{01}\left(\theta_{0}\right)$. In the $45^{\circ} / 0^{\circ}$ measuring geometry, the collimated incident light reaches the interface from the air side at angle of $45^{\circ}$. For an interface having a relative index of refraction of 1.5 , we have $R_{01}\left(\theta_{0}\right)=0.05$ and therefore $T_{01}\left(\theta_{0}\right)=0.95$.

When the incident light illuminates the print side of the interface under an incidence $\theta_{1}$, the interface's reflectance and transmittance are, respectively, $R_{10}\left(\theta_{1}\right)$ and $T_{10}\left(\theta_{1}\right)=1-R_{10}\left(\theta_{1}\right)$. Calling $\theta_{1}^{\prime}$ the angle of emergence of the light into the air, related with $\theta_{1}$ according to Snell's refraction law, the transmittance of the interface is also $T_{10}\left(\theta_{1}^{\prime}\right)=T_{01}\left(\theta_{1}\right)$. In the $45^{\circ} / 0^{\circ}$ measuring geometry, the detector captures the light emerging into the air perpendicularly to the print $\left(\theta_{1}=\theta_{1}^{\prime}=0^{\circ}\right)$. For a relative index of refraction of 1.5 , we have $R_{10}\left(0^{\circ}\right)=0.04$ and therefore $T_{10}\left(\theta_{1}\right)=0.96$.

When the incident light is Lambertian, the interface's reflectance and transmittance are, respectively, $r_{01}$ [Eq. (15)] and $t_{01}$ [Eq. (16)] for an illumination at the air side and $r_{10}$ [Eq. (18)] and $t_{10}$ [Eq. (19)] for an illumination at the print side. According to Eqs. (17), (19), and (20), we have $t_{01}=1-r_{01}, t_{10}=1-r_{10}$, and $t_{10}=\left(n_{0} / n_{1}\right)^{2} t_{01}$. For a relative index of refraction of 1.5 , we have $r_{01}=0.092, t_{01}$ $=0.908, r_{10}=0.596$, and $t_{10}=0.404$. For other refractive indices, evaluation tables of $r_{01}$ and $r_{10}$ have been published by Judd. ${ }^{24}$ The diffuse reflectance of a colored interface is a function of the transmittance $t$ of the colorant layer. It is expressed by Eq. (22). Table 2 gives numerical values of $r(t)$ as a function of $t$ for a print-air relative refractive index of 1.5. A linear interpolation of these values provides a good approximation of the exact function $r(t)$.

Table 2. Diffuse Reflectance of Colored Interfaces ${ }^{a}$

\begin{tabular}{llllll}
\hline \multicolumn{1}{c}{$t$} & $r(t)$ & \multicolumn{1}{c}{$t$} & $r(t)$ & \multicolumn{1}{c}{$t$} & $r(t)$ \\
\hline 0 & 0 & 0.5 & 0.047 & 0.83 & 0.272 \\
0.15 & 0.001 & 0.55 & 0.065 & 0.87 & 0.327 \\
0.25 & 0.006 & 0.6 & 0.086 & 0.9 & 0.374 \\
0.3 & 0.01 & 0.65 & 0.113 & 0.93 & 0.429 \\
0.35 & 0.016 & 0.7 & 0.146 & 0.95 & 0.47 \\
0.4 & 0.023 & 0.75 & 0.187 & 0.97 & 0.516 \\
0.45 & 0.034 & 0.8 & 0.237 & 1 & 0.596 \\
\hline
\end{tabular}

${ }^{a}$ As a function of ink normal transmittance $t$ for a relative refractive index of 1.5.

\section{APPENDIX B: AVERAGE PATH $\mu$ TRAVERSED BY THE DIFFUSE INCIDENT LIGHT IN THE INKED LAYER}

Incident diffuse light crossing a colored interface is attenuated by the factor $T_{\text {in }}$ expressed in Section 3 [Eq. (27)]. In approximation (28), we propose a simplified expression where the coefficient $\mu$ represents the average path traversed by the light within the inked layer. The approximation is

$$
T_{\mathrm{in}}=\int_{\theta=0}^{\pi / 2} T_{01}(\theta) t^{\mu(\theta)} \sin 2 \theta \mathrm{d} \theta \approx t^{\mu} \int_{\theta=0}^{\pi / 2} T_{01}(\theta) \sin 2 \theta \mathrm{d} \theta,
$$

where the function $\mu(\theta)$ is given by Eq. (24), with $t$ being the normal transmittance of the ink layer.

On the left side of Eq. (B1), the exact expression of $T_{\text {in }}$ depends on the Fresnel coefficient $T_{01}(\theta)$, which is a function of the relative refractive index $n=n_{1} / n_{0}$ of the printair interface and on the transmittance $t$ of the inked layer at normal incidence. On the right side, the expression of $T_{\text {in }}$ is decomposed into a term $t^{\mu}$ depending only on the ink transmittance, and an integral, equal to the external transmittance of the interface $t_{01}$ [Eq. (16)], depending only on the relative refractive index of the print-air interface. Typically, the print-air interface has a relative refractive index of 1.5 and $t_{01}$ is equal to 0.9 for diffuse incident light.

The exponent $\mu$ applied to the ink transmittance is a constant corresponding to the average oblique path traversed by the diffuse light within the inked layer. If the irradiance crossing the inked layer would have been Lambertian, all the oblique paths within the inked layer would have been traversed by the same radiance. But since the Fresnel transmission coefficient is angle dependent, the part of the diffuse incident light transmitted by the interface is not Lambertian. The oblique paths within the inked layer are therefore traversed by radiances that depend on the Fresnel transmission coefficient, and the average oblique path $\mu$ traversed by the diffuse light therefore depends on the refractive index of the print-air interface.

Let us numerically compute the coefficient $\mu$ for a given refractive index. We take $m$ equidistant values $t_{i}$ of the inked layer's transmittance, from $t_{0}=0$ to $t_{m-1}=1$. We look for the coefficient $\mu$ that minimizes the sum of the square differences between the exact expression of $T_{\text {in }}$ and its approximated expression, i.e., the coefficient $\mu$ that minimizes

$$
\sum_{i=0}^{m-1}\left(t_{i}^{\mu} t_{01}-\int_{\theta=0}^{\pi / 2} T_{01}(\theta) t_{i}^{\mu(\theta)} \sin 2 \theta \mathrm{d} \theta\right)^{2} .
$$

For a print-air refractive index of 1.5 , we obtain $\mu$ $=1.134$. Equation (B1) is very accurate. If we plot the exact and the approximated expressions of $T_{\text {in }}$ as functions of $t_{i}$ on the same graph, both curves are perfectly superposed. 


\section{APPENDIX C: SOLVING THE KUBELKA-MUNK DIFFERENTIAL EQUATION SYSTEM WITH THE LAPLACE TRANSFORM}

Let us solve the Kubelka-Munk differential equation system

$$
\begin{aligned}
& \frac{\mathrm{d}}{\mathrm{d} x} i_{r}(x)=(K+S) i_{r}(x)-S i_{t}(x), \\
& \frac{\mathrm{d}}{\mathrm{d} x} i_{t}(x)=S i_{r}(x)-(K+S) i_{t}(x) .
\end{aligned}
$$

To convert this differential equation system into a linear system, we apply the Laplace transform, ${ }^{31}$ which transforms a causal function $f(x)$ into a function $F(p)$ defined by

$$
F(p)=\int_{0}^{\infty} f(t) e^{-p t} \mathrm{~d} t .
$$

The Laplace transform is linear, i.e., $a f(x)+b g(x)$ is transformed into $a F(p)+b G(p)$. The derivative of function $f$ is transformed into $p F(p)-f(0)$, where the constant $f(0)$ is the value of $f$ at $x=0$.

Let us apply the Laplace transform to the equation system $(\mathrm{C} 1)$. We call $I_{r}(p)$ and $I_{t}(p)$ the transforms of $i_{r}(x)$ and $i_{t}(x)$. We obtain the following linear system of two equations with the two unknown variables $I_{r}(p)$ and $I_{t}(p)$ :

$$
\begin{aligned}
& p I_{r}(p)-i_{r}(0)=(K+S) I_{r}(p)-S I_{t}(p), \\
& p I_{t}(p)-i_{t}(0)=S I_{r}(p)-(K+S) I_{t}(p) .
\end{aligned}
$$

The solutions of the linear equation system (C2) are

$$
\begin{aligned}
& I_{r}(p)=\frac{i_{r}(0)(p+a S)-S i_{t}(0)}{p^{2}-b^{2} S^{2}}, \\
& I_{t}(p)=\frac{i_{t}(0)(p-a S)+S i_{r}(0)}{p^{2}-b^{2} S^{2}},
\end{aligned}
$$

with

$$
\begin{aligned}
& a=(K+S) / S, \\
& b=\sqrt{a^{2}-1},
\end{aligned}
$$

and where $i_{r}(0)$ is the downward irradiance located at the recto boundary of the layer, i.e., at the depth $x=0$, and $i_{t}(0)$ is the upward irradiance located at the verso boundary, i.e., at depth $x=h$.

The terms $p /\left(p^{2}-b^{2} S^{2}\right)$ and $b S /\left(p^{2}-b^{2} S^{2}\right)$ are, respectively, the transforms of $\cosh (b S x)$ and $\sinh (b S x)$. Therefore, Eqs. (C3) and (C4) are the transforms of functions $i_{r}(x)$ and $i_{t}(x)$ expressed as

$$
i_{r}(x)=i_{r}(0) \cosh (b S x)+\frac{1}{b}\left(a i_{r}(0)-i_{t}(0)\right) \sinh (b S x),
$$

$$
i_{t}(x)=i_{t}(0) \cosh (b S x)+\frac{1}{b}\left(i_{r}(0)-a i_{t}(0)\right) \sinh (b S x) .
$$

Equations (C5) and (C6) are the general solutions of the Kubelka-Munk differential equation system (C1), where the downward and upward irradiances $i_{r}(0)$ and $i_{t}(0)$ at the recto boundary $x=0$ depend on the exterior illumination and on the recto and verso reflectors.

\section{APPENDIX D: DERIVING THE REFLECTANCE AND THE TRANSMITTANCE OF A SCATTERING LAYER BOUNDED BY TWO REFLECTORS FROM THE KUBELKA-MUNK DIFFERENTIAL EQUATION SYSTEM}

The internal reflectance and internal transmittance of the scattering layer are given, respectively, by the ratios $i_{r}(0) / I_{0}$ and $i_{t}(h) / I_{0}$. The expressions of $i_{r}(0)$ and $i_{t}(h)$ are derived from the general solutions of Eqs. (C5) and (C6) of the Kubelka-Munk differential equation system (C1) by applying specific boundary conditions.

Let us consider a layer bounded at $x=0$ and $x=h$ by two reflectors of respective reflectances $r_{0}$ and $r_{h}$. These reflectors may be interfaces between two media, interfaces incorporating an inked layer, or backscattering backgrounds.

At $x=0$, the layer receives the source irradiance $I_{0}$ downwards. In addition to this source irradiance, a fraction $r_{0}$ of the upward irradiance $i_{r}(0)$ is reflected by the recto and also propagates downwards. The total downward irradiance $i_{t}(0)$ is therefore

$$
i_{t}(0)=I_{0}+r_{0} i_{r}(0) .
$$

At $x=h$, the sublayer receives the downward irradiance $i_{t}(h)$. There is no light propagating upwards from the exterior. However, a fraction $r_{h}$ of the irradiance $i_{t}(h)$ is reflected by the lower reflector and forms the upward irradiance $i_{r}(h)$ :

$$
i_{r}(h)=r_{h} i_{t}(h) .
$$

We can insert the expression for $i_{r}(h)$ given by Eq. (D2) at $x=h$ into Eq. (C5):

$$
\begin{aligned}
r_{h} i_{t}(h)= & i_{r}(0) \cosh (b S h)+\frac{1}{b}\left[a i_{r}(0)-\left(I_{0}\right.\right. \\
& \left.\left.+r_{0} i_{r}(0)\right)\right] \sinh (b S h) .
\end{aligned}
$$

We insert into Eq. (C6) the expression for $i_{t}(0)$ given by Eq. (D1):

$$
\begin{aligned}
i_{t}(h)= & \left(I_{0}+r_{h} i_{r}(0)\right) \cosh (b S h)+\frac{1}{b}\left[i_{r}(0)-a\left(I_{0}\right.\right. \\
& \left.\left.+r_{0} i_{r}(0)\right)\right] \sinh (b S h) .
\end{aligned}
$$

Equations (D3) and (D4) form a linear system of two equations depending on the two unknown variables $i_{r}(0)$ and $i_{t}(h)$, i.e., on the two irradiances exiting the print. The solutions of this system are 


$$
\begin{aligned}
i_{r}(0) & =I_{0} \frac{\left(1-a r_{h}\right) \sinh (b S h)+b r_{h} \cosh (b S h)}{\left(a-r_{0}-r_{h}+a r_{0} r_{h}\right) \sinh (b S h)+b\left(1-r_{0} r_{h}\right) \cosh (b S h)}, \\
i_{t}(h) & =I_{0} \frac{b}{\left(a-r_{0}-r_{h}+a r_{0} r_{h}\right) \sinh (b S h)+b\left(1-r_{0} r_{h}\right) \cosh (b S h)} .
\end{aligned}
$$

The ratios $i_{r}(0) / I_{0}$ and $i_{t}(h) / I_{0}$ are, respectively, the print's internal reflectance $R_{b}$ and transmittance $T_{b}$ that include the multiple reflections at the upper and the lower reflectors $\left(r_{0}\right.$ and $\left.r_{h}\right)$ :

$$
R_{b}=\frac{\left(1-a r_{h}\right) \sinh (b S h)+b r_{h} \cosh (b S h)}{\left(a-r_{0}-r_{h}+a r_{0} r_{h}\right) \sinh (b S h)+b\left(1-r_{0} r_{h}\right) \cosh (b S h)},
$$

$$
T_{b}=\frac{b}{\left(a-r_{0}-r_{h}+a r_{0} r_{h}\right) \sinh (b S h)+b\left(1-r_{0} r_{h}\right) \cosh (b S h)} .
$$

\section{APPENDIX E: ORIGINAL KUBELKA-MUNK MODEL AND SAUNDERSON CORRECTION}

In their original papers, ${ }^{1,7}$ Kubelka and Munk consider a substrate layer superposed on a background of reflectance $r_{g}$ having the same refractive index as the substrate layer. The recto interface is ignored. According to Kubelka, [Ref. 7, Eq. 26], the reflectance $R_{\mathrm{KM}}$ of the substrate bounded by the background is

$$
R_{\mathrm{KM}}=\frac{\left(1-a r_{g}\right) \sinh (b S h)+b r_{g} \cosh (b S h)}{\left(a-r_{g}\right) \sinh (b S h)+b \cosh (b S h)} .
$$

Equation (E1) can be derived from Eq. (D7) by setting $r_{0}=0$ and $r_{h}=r_{g}$. However, in the case where the substrate, typically of refractive index 1.5 , is in contact with the air at the recto, the recto interface cannot be ignored since it behaves as a reflector. A strongly scattering layer illuminates the interface with air with Lambertian irradiance. The reflectance of the interface is therefore $r_{10}$. Saunderson ${ }^{9}$ introduced a correction to the KubelkaMunk reflectance [Eq. (E1)] accounting for the optical effects of the recto interface, i.e., the surface specular reflection (ignored when the incidence angle and the observation angle are different), the penetration of light through the interface (attenuation $T_{\text {in }}$ ), the emergence of light through the interface (attenuation $T_{\mathrm{ex}}$ ), and the multiple reflections occurring between the scattering layer (reflectance $R_{\mathrm{KM}}$ expressed according to the KubelkaMunk model) and the interface (reflectance $r_{10}$ ):

$$
R_{S}=T_{\mathrm{in}} T_{\mathrm{ex}} \frac{R_{\mathrm{KM}}}{1-r_{10} R_{\mathrm{KM}}} .
$$

$R_{S}$ expresses the global reflectance of the bounded scattering layer when the surface specular reflection is discarded. Let us expand the expression of $R_{S}$ by replacing in Eq. (E2) the term $R_{\mathrm{KM}}$ by its expression in Eq. (E1). The resulting expression matches the expression of Eq. (D7) of $R_{b}$ obtained by considering directly the scattering medium bounded at the recto and the verso by two reflectors of respective reflectances $r_{0}=r_{10}$ and $r_{h}=r_{g}$. Therefore, the Saunderson-corrected Kubelka-Munk model is a special form of our extended Kubelka-Munk formulation [Eq. (D7)] for a scattering medium bounded by two reflectors of respective reflectances $r_{0}$ and $r_{h}$.

\section{ACKNOWLEDGMENT}

We thank Jean-Marie Becker of Ecole Supérieure de Chimie-Physique-Electronique de Lyon and Laboratoire de Traitement du Signal et Instrumentation, SaintEtienne, for fruitful discussions about the Laplace transform.

M. Hébert's e-mail address is mathieu.hebert@epfl.ch.

\section{REFERENCES}

1. P. Kubelka and F. Munk, "Ein Beitrag zur Optik der Farbanstriche," Z. Tech. Phys. (Leipzig) 12, 593-601 (1931)

2. H. E. J. Neugebauer, "Die theoretischen Grundlagen des Mehrfarbendrucks," Z. Wiss. Photog. 36, 73-89 (1937); reprinted in Neubegauer Memorial Seminar on Color Reproduction, K. Sayanasi, ed., Proc. SPIE 1184, 194-202 (1989).

3. J. A. C. Yule and W. J. Nielsen, "The penetration of light into paper and its effect on halftone reproductions," in Proceedings of the Technical Association of the Graphic Arts (TAGA, 1951), Vol. 3, pp. 65-76.

4. J. A. S. Viggiano, "Modeling the color of multi-colored halftones," in Proceedings of the Technical Association of the Graphic Arts (TAGA, 1990), pp. 44-62.

5. F. R. Clapper and J. A. C. Yule, "Effect of multiple internal reflections on the densities of halftone prints on paper," J. Opt. Soc. Am. 43, 600-603 (1953).

6. F. C. Williams and F. R. Clapper, "Multiple internal reflections in photographic color prints," J. Opt. Soc. Am. 43, 595-599 (1953).

7. P. Kubelka, "New contributions to the optics of intensely light-scattering material. Part I," J. Opt. Soc. Am. 38, 448-457 (1948).

8. P. Kubelka, "New contributions to the optics of intensely light-scattering materials. Part II: Nonhomogeneous layers," J. Opt. Soc. Am. 44, 330-335 (1954).

9. J. L. Saunderson, "Calculation of the color pigmented plastics,” J. Opt. Soc. Am. 32, 727-736 (1942).

10. J. S. Arney, "A probability description of the Yule-Nielsen effect: I," J. Imaging Sci. Technol. 41, 633-636 (1997).

11. G. Rogers, "Effect of light scatter on halftone color," J. Opt. Soc. Am. A 15, 1813-1821 (1998).

12. L. Yang, R. Lenz, and B. Kruse, "Light scattering and ink penetration effects on tone reproduction," J. Opt. Soc. Am. A 18, 360-366 (2001)

13. G. Rogers, "A generalized Clapper-Yule model of halftone reflectance," Color Res. Appl. 25, 402-407 (2000). 
14. R. D. Hersch, P. Emmel, F. Collaud, and F. Crété, "Spectral reflection and dot surface prediction models for color halftone prints," J. Electron. Imaging 14, 33001-12 (2005).

15. M. Hébert and R. D. Hersch, "Extending the Clapper-Yule model to rough printing supports," J. Opt. Soc. Am. A 22, 1952-1967 (2005).

16. P. Emmel and R. D. Hersch, "A unified model for color prediction of halftoned prints," J. Imaging Sci. Technol. 44, 351-359 (2000).

17. L. Yang and B. Kruse, "Revised Kubelka-Munk theory. I. Theory and application," J. Opt. Soc. Am. A 21, 1933-1941 (2004).

18. L. Yang and B. Kruse, "Revised Kubelka-Munk theory. II. Unified framework for homogeneous and inhomogeneous optical media," J. Opt. Soc. Am. A 21, 1942-1952 (2004).

19. L. Yang and B. Kruse, "Revised Kubelka-Munk theory. III. A general theory of light propagation in scattering and absorptive media," J. Opt. Soc. Am. A 22, 1866-1873 (2005).

20. S. Chandrasekhar, Radiative Transfer (Dover, 1960).

21. W. E. Vargas and G. A. Niklasson, "Applicability conditions of the Kubelka-Munk theory,” Appl. Opt. 36, 5580-5586 (1997).
22. M. Born and E. Wolf, Principle of Optics, 7th expanded ed. (Pergamon, 1999), p. 47.

23. W. R. McCluney, Introduction to Radiometry and Photometry (Artech, 1994), pp. 7-13.

24. D. B. Judd, "Fresnel reflection of diffusely incident light," J. Res. Natl. Bur. Stand. 29, 329-332 (1942).

25. H.-H. Perkampus, Encyclopedia of Spectroscopy (VCH, 1995).

26. J. D. Shore and J. P. Spoonhower, "Reflection density in photographic color prints: generalizations of the Williams-Clapper transform," J. Imaging Sci. Technol. 45 , 484-488 (2001).

27. H. Hébert and R. D. Hersch, "Classical print reflection models: a radiometric approach," J. Imaging Sci. Technol. 48, 363-374 (2004).

28. M. E. Demichel, Procédé, 26, 17-21 (1924), see also Ref. 29

29. D. R. Wyble and R. S. Berns, "A critical review of spectral models applied to binary color printing," Color Res. Appl. 25, 4-19 (2000).

30. G. Sharma, "Color fundamentals for digital imaging," in Digital Color Imaging Handbook, G. Sharma, ed. (CRC Press, 2003), pp. 1-114.

31. J. W. Harris and H. Stocker, Handbook of Mathematics and Computational Science (Springer-Verlag, 1998), pp. 736-758. 